\title{
INTEGRABLE SYSTEMS AND GROUP ACTIONS
}

\author{
EVA MIRANDA
}

\begin{abstract}
The main purpose of this paper is to present in a unified approach to see different results concerning group actions and integrable systems in symplectic, Poisson and contact manifolds. Rigidity problems for integrable systems in these manifolds will be explored from this perspective.
\end{abstract}

\section{Contents}

1. Introduction 1

2. The Symplectic case 4

2.1. Action-angle coordinates: Torus actions meet integrable systems 4

$\begin{array}{ll}\text { 2.2. Singular action-angle coordinates } & 7\end{array}$

2.3. Action-angle coordinates, group actions and rigidity 19

3. The Contact case 20

3.1. Contact manifolds and integrability 22

3.2. Additional symmetries and rigidity 27

4. The Poisson case 29

4.1. Motivating examples 30

4.2. A Darboux-Carathéodory theorem in the Poisson context 34

4.3. Split systems and rigidity 34

4.4. An action-angle theorem for Poisson manifolds 35

4.5. Equivariant theorems for Poisson manifolds and rigidity phenomena 36

References $\quad 37$

\section{INTRODUCTION}

From the very beginning: symmetries, group actions and integrable systems have been close allies. The study of symmetries of the differential systems given

Date: August 29, 2012.

1991 Mathematics Subject Classification. 53D17.

Key words and phrases. integrable system, momentum map, Poisson manifold, Contact manifold, symplectic manifold, group action.

Partially supported by the DGICYT/FEDER project MTM2009-07594: Estructuras Geometricas: Deformaciones, Singularidades y Geometria Integral. 
by Hamilton's equation associated to an energy function $H$, led naturally to the existence of Poisson commuting functions and the method of integration by quadratures. When the number of commuting functions is maximal, the resolution by quadratures is possible and the set of commuting functions defines an integrable system on the phase space $T^{*}\left(\mathbb{R}^{n}\right)$. The idea can be naturally exported to any symplectic manifold and the reduction process can be seen as a reduction of the dynamics in the manifolds given by a Marsden-Weinstein reduction associated to a toric group.

One of the most striking and basic results in the theory of integrable systems on symplectic manifolds is the theorem of Liouville-Mineur-Arnold [46],[47],[2] which states that the foliation defined by a regular integrable system in the neighbourhood of a compact fibre is a fibration by tori and that the symplectic form can be given semilocally as a Darboux form. This theorem is achieved by constructing actionangle coordinates in a neighbhourhood of a regular fibre and proving a Darboux theorem in this coordinates. It is really enriching to understand and interpret the difficulties in extending this theorem to a more general context. This is the case of including singularities into the picture or trying to construct global action-angle coordinates. The study of these problems concerning integrable systems is still a challenging issue for symplectic topologists, geometers and dynamicists.

Experts working in dynamical systems are interested in the properties of the Hamiltonian vector field and its flow. Topologists are interested in both constructing global examples of integrable systems and studying obstruction theories. Geometers are interested in understanding the geometrical structure (symplectic) of these objects and in proving classification theorems.

Symmetries are present in many physical problems and therefore they show up in integrable systems theory as well. The ace in the hole in the study of integrable systems is to look for symmetries. The very proof of Liouville-Mineur-Arnold uses this strategy. It finds a toric Hamiltonian action tangent to the fibres of the moment map. In this paper we will also consider additional symmetries showing up. We will try to study which properties hold for these additional symmetries. Those symmetries are encoded in actions of Lie groups.

Among all kind of symmetries the toric ones play a central rôle in this paper. Hamiltonian actions of tori in symplectic geometry have attracted the attention of many specialists. Along the way many results of symplectic uniqueness are obtained. A good example of this is Delzant's theorem [15] which enables to recover information of a compact $2 n$-dimensional manifold by looking at the image of the moment map of a Hamiltonian torus action which is a convex polytope in $\mathbb{R}^{n}$. A lot of contributions in the area of Hamiltonian actions of Lie groups have been done ever since. Let us mention some of the references of the large list of results in that direction: the works of Lerman and Tolman to extend those result to symplectic orbifolds ([39]) and the works of Karshon and Tolman for complexity one Hamiltonian group actions ([31]) among many others. One of the current topics of interest are singularities of integrable systems. Besides the classical references on the symplectic geometry and topology of these singularities [21], [20], [45], [6], [77], and more recently the work in connection to (semi)-toric actions and singularities ([64], [65]). 
The notion of integrability and its connection to group actions can be naturally studied in the contact context too. Toric contact manifolds have been largely studied by Banyaga and Molino [3, 4], Luzt [43] and Lerman [38]. In [49] the integrable non-degenerate but not necessarily toric case is studied. More recently, integrability in the contact context has been studied by Khesin and Tabaschnikov [16]. We will present classification results for integrable systems with non-degenerate singularities in contact manifolds and give complete proofs.

Last but not least, Poisson manifolds constitute a natural scenario to study Hamilton's equations. As a first and basic example of Poisson manifolds, we have the dual of a Lie algebra. Poisson manifolds are foliated by symplectic manifolds but this foliation is not necessarily regular. When considering a Hamiltonian system in a Poisson manifold we obtain families of Hamiltonian systems in the symplectic leaves of this foliation but there are some additional "transversal" structures given by extra symmetries (Casimir functions). We can therefore define integrable systems on these manifolds too and study similar topological/geometrical and dynamical properties.

\section{Organization of this paper:}

This paper is divided in three sections: The symplectic, contact and Poisson section. In this paper we wanted to give a global perspective and underline a common strategy in symplectic, contact and Poisson about the role of group actions in studying integrable systems: their (equivariant) normal and also some rigidity issues.

Section 2 is mainly a recompilation of results about symplectic linearization of singular Lagrangian foliations defined by integrable systems contained in [49], [58].

We include here an outline of the proof of symplectic linearization. A more detailed proof can be found in [49], [58], [54]. We also re-state Eliasson's theorem using the foliation defined by the Hamiltonian vector fields. This formulation is necessary since the existence of hyperbolic singularities one cannot guarantee that the moment map is a function of the elements in Williamson's basis.

In this section we also describe some applications to action-angle coordinates to the context of Geometric Quantization and we interconnect some results of rigidity of group actions with the symplectic linearization results to obtain structural stability of the foliation.

Section 3 contains result about equivalence with what we call "linear models" for integrable systems in the contact case: We include here complete proofs of contact equivalence with the contact models. This result was announced in a short note before [50] but contained no proofs. We offer here an improved version of the proof contained in [49] which makes special emphasis on the tools of group actions.

Section 4 contains results about action-angle coordinates for integrable systems and rigidity for Hamiltonian actions on Poisson manifolds. Most of the results contained in the Poisson case are joint results with other coauthors and the complete proof of the statements is contained in [37] and [56]. The results are presented as a summary of old results with a new perspective. We focus on the rôle of actions and symmetries in the proofs. We also present new examples of integrable system in the Poisson context. As a bonus we also prove that, unlike the symplectic and contact case, integrable systems on Poisson manifolds are not rigid. 


\section{The Symplectic Case}

In June 29th of 1853 Joseph Liouville presented a communication entitled "Sur l'intégration des equations différentielles de la Dynamique" at the "Bureau des longitudes". In the resulting note [42] the notion of integrability of the system is related to the existence of $n$ integrals in involution with respect to the Poisson bracket attached to the symplectic form. These systems come into the scene with the classical denomination of "completely integrable systems".

An integrable system on a symplectic manifold $\left(M^{2 n}, \omega\right)$ is given by a set of generically independent functions $F=\left(f_{1}, \ldots, f_{n}\right)$ satisfying $\left\{f_{i}, f_{j}\right\}=0, \forall i, j$. The mapping $F: M^{2 n} \longrightarrow R^{n}$ given by $F=\left(f_{1}, \ldots, f_{n}\right)$ is called moment map.

The distribution generated by the Hamiltonian vector fields $X_{f_{i}}$ is involutive. It spans an integrable distribution which is of maximal rank at points where the functions are functionally independent. In this case, the leaf of the foliation integrating the distribution is Lagrangian since $0=\left\{f_{1}, f_{j}\right\}=\omega\left(X_{f_{i}}, X_{f_{j}}\right)$ and tangent to the fibers of $F=\left(f_{1}, \ldots, f_{n}\right)$. When we consider a point where the functions are not functionally independent the leaf of the foliation is isotropic.

We are interested in understanding the geometry of integrable systems on symplectic manifolds. On the one hand, we are interested in classifying the symplectic structures which make a fixed integrable system into a Lagrangian foliation. On the other hand we want to find "normal forms" for the fibration defined by $\mathbb{F}$. In this paper we address the generic case. Namely, we consider the regular and the non-degenerate singular case.

2.1. Action-angle coordinates: Torus actions meet integrable systems. In this section we will assume that the moment map is proper. Let $L$ be a regular orbit of this distribution then by the discussion above, this orbit is a Lagrangian submanifold. It was Liouville [42] who first observed that these Lagrangian submanifolds are indeed tori. Later, Mineur [46],[47] realized that the neighbouring orbits are also tori and the fibration can be given via "action" coordinates in a neighbhourhood of a regular fiber (called Liouville tori). This is a topological consequence of the theorem of Liouville-Mineur-Arnold ${ }^{1}$ which we state below. The geometrical contribution of the ensures the existence of symplectic normal forms in the neighbourhood of a compact regular orbit.

\section{Theorem 2.1. (Liouville-Mineur-Arnold Theorem)}

Let $\left(M^{2 n}, \omega\right)$ be a symplectic manifold and let $F: M^{2 n} \longrightarrow \mathbb{R}^{n}$ be a proper moment map. Assume that the components $f_{i}$ of $F$ are pairwise in involution with respect to the Poisson bracket associated to $\omega$ and that $d f_{1} \wedge \cdots \wedge d f_{n} \neq 0$ on a dense set. Let $N=F^{-1}(c), c \in \mathbb{R}^{n}$ be a connected regular levelset. Then there exists a neighbourhood $U(L)$ of $L$ and a diffeomorphism $\phi: U(L) \longrightarrow D^{n} \times \mathbb{T}^{n}$ such that,

(1) $\phi(L)=\{0\} \times \mathbb{T}^{n}$.

(2) A set of coordinates $\mu_{i}$ in $D^{n}$ and a set of coordinates $\beta_{i}$ in $\mathbb{T}^{n}$ for which, $\phi^{*}\left(\sum_{i=1}^{n} d \mu_{i} \wedge d \beta_{i}\right)=\omega$.

\footnotetext{
${ }^{1}$ This theorem is classically known as Arnold-liuville theorem. To the author's knowledge, the works of Henri Mineur [46, 47] already gave the a complete description of the Hamiltonian system in a neighbourhood of a compact regular orbit. That is why we will refer to the classical Arnold-Liouville theorem as Liouville-Mineur-Arnold theorem.
} 
(3) $F$ depends only on $\phi^{*}\left(\mu_{i}\right)=p_{i}$ and it does not depend on $\phi^{*}\left(\beta_{i}\right)=\theta_{i}$.

The new coordinates $p_{i}$ obtained are called action coordinates. The coordinates $\theta_{i}$ are called angle coordinates. Mineur also showed that the action functions $p_{i}$ can be defined via the period integrals. Let $x$ be a point in a small neighbourhood of $L$, the period integrals are defined as:

$$
p_{i}(x)=\int_{\Gamma_{i}(x)} \alpha
$$

where $\alpha$ is a Liouville one-form $(d \alpha=\omega)$, and $\Gamma_{i}(x)$ is a closed curve which depends smoothly on $x$ and which lies on the Liouville torus containing $x$. The homology classes of $\Gamma_{1}(x), \ldots, \Gamma_{n}(x)$ form a basis of the first homology group of the Liouville torus.

The existence of action-angle coordinates in a neighbourhood of a compact orbit provides a symplectic model for the Lagrangian foliation $\mathcal{F}$ determined by the Hamiltonian vector fields of the $n$ component functions $f_{i}$ of the moment map $F$. As a matter of fact, Liouville-Mineur-Arnold theorem entails a "uniqueness" result for the symplectic structures making the foliation $\mathcal{F}$ into a Lagrangian foliation. Namely, if $\omega_{1}$ and $\omega_{2}$ are two symplectic structures defined in a neighbourhood of $L$ for which $\mathcal{F}$ is Lagrangian then there exists a symplectomorphism preserving the foliation, fixing $L$ and carrying $\omega_{1}$ to $\omega_{2}$. This is due to the following observation: Let $X_{f_{i}}$ be the Hamiltonian vector fields associated to the functions $f_{i}$ for any $1 \leq i \leq n$, then the Lagrangian condition implies that $\mathcal{F}=<X_{f_{1}}, \ldots, X_{f_{n}}>$, further $\left\{f_{j}, f_{k}\right\}_{i}=0$ where $\{., .\}_{i}$ stands for the Poisson bracket attached to $\omega_{i}$ $, i=1,2$. Then by virtue of Liouville-Mineur-Arnold theorem there exists a foliation-preserving symplectomorphism $\phi_{i}$ taking $\omega_{i}$ to $\omega_{0}=\sum_{i=1}^{n} d p_{i} \wedge d \theta_{i}$. In all, the diffeomorphism $\phi_{2}^{-1} \circ \phi_{1}$ does the job. It takes $\omega_{1}$ to $\omega_{2}$, it fixes $L$ and it is foliation preserving.

So if the orbit is regular the existence of action-angle coordinates enables to classify the symplectic germs, up to foliation-preserving symplectomorphism, for which $\mathcal{F}$ is Lagrangian in a neighbourhood of a compact orbit. There is just one class of symplectic germs for which the foliation is Lagrangian and the problem of classification as Lagrangian fibrations or integrable systems is the same.

One could look at the problem from a global perspective. There are topological obstructions to the existence of global action-angle coordinates as it was shown by Duistermaat in [19].

\section{Consequences:}

Action-angle coordinates give:

- An action of a torus $\mathbb{T}^{n}$ tangent to the Liouville tori which is Hamiltonian.

- A normal form for the set of first integrals in involution (action coordinates). In these coordinates, $F=\left(p_{1}, \ldots, p_{n}\right)$.

- A normal form for the symplectic structure: the symplectic structure is Darboux $\omega=\sum_{i=1}^{n} d p_{i} \wedge d \theta_{i}$.

Remark 2.2. Indeed one of the author's favourite proof of the action-angle theorem is in the paper of Duistermaat [19]. The theorem uses strongly the existence of a 
torus action tangent to the fibration given by the moment map to construct actionangle coordinates. This idea can be exported to the regular Poisson case as we will see later.

2.1.1. Applications of action-angle coordinates. The global problem of existence of action-angle variables is related to Monodromy and the Chern class of the fibration given by the fibers of the moment map.

In the case of generalized global action-angle coordinates on compact manifolds, the semi-local torus action extend to a torus action $\mathbb{T}^{n}$ on the compact symplectic manifolds $\left(M^{2 n}, \omega\right)$. We get global action-angle coordinates with singularities and a toric manifold. Symplectic geometry can be read from the Delzant polytope [15].

The existence of action-angle coordinates has many implications in dynamics: for instance the topological entropy of these systems is zero if there are no singularities in the way [63] and [35].

Other applications of global action-angle coordinates show up (not so unexpectedly) in the context of geometric quantization using real polarizations. We include here a short summary. A more extended version of these applications (with singularities in the way) can be found in the short note [53].

Let $\left(M^{2 n}, \omega\right)$ be a symplectic manifold such that $[\omega]$ is integral. Under these circumstances (see for instance [73] ), there exists a complex line bundle $\mathbb{L}$ with a connection $\nabla$ over $M$ such that $\operatorname{curv}(\nabla)=\omega$. The symplectic manifold $\left(M^{2 n}, \omega\right)$ is called prequantizable and the pair $(\mathbb{L}, \nabla)$ is called a prequantum line bundle of $\left(M^{2 n}, \omega\right)$. In order to construct the geometric quantization of these objects, we need to restrict the space of sections to a subspace of sections which are flat in "priviledged" directions given by a polarization. A real polarization is a Lagrangian foliation. In the case the polarizations are given by integrable systems, there is a connection between well-defined flat sections and action-angle coordinates. Consider the following:

Example 2.3. Consider $M=S^{1} \times \mathbb{R}$ and $\omega=d t \wedge d \theta$. Take as $\mathbb{L}$ the trivial bundle with connection 1 -form $\Theta=t d \theta$. Now, let $\mathcal{P}=<\frac{\partial}{\partial \theta}>$ then flat sections satisfy, $\nabla_{X} \sigma=X(\sigma)-i<\theta, X>\sigma=0$ Thus flat sections $\sigma(t, \theta)=a(t) . e^{i t \theta}$ are defined along leaves are given by the condition $t=2 \pi k, k \in \mathbb{Z}$.

This example shows that flat sections are not globally defined but they exist along a subset of leaves of the polarization. These are called Bohr-Sommerfeld leaves. The characterization of Bohr-Sommerfeld leaves for regular fibrations under some conditions is a well-known result by Guillemin and Sternberg ([27]). In particular the set of Bohr-Sommerfeld leaves is discrete and is given by "action" coordinates.

Theorem 2.4 (Guillemin-Sternberg). If the polarization is a regular fibration with compact leaves over a simply connected base B, then the Bohr-Sommerfeld set is discrete and assuming that the zero-fiber is a Bohr-Sommerfeld leaf, the BohrSommerfeld set is given by, $B S=\left\{p \in M,\left(f_{1}(p), \ldots, f_{n}(p)\right) \in \mathbb{Z}^{n}\right\}$ where $f_{1}, \ldots, f_{n}$ are global action coordinates on $B$.

This result connects with Liouville-Mineur-Arnold theorem. When we consider a toric manifolds the base $B$ may be identified with the image of the moment map by the toric action (Delzant polytope). 
In view of the previous theorem, when the polarization is given by an integrable system with global action-angle coordinates it makes sense to "quantize" these systems counting integral Liouville tori.

This can be formalized following the idea of Kostant [34], in the case there are no global sections denote by $\mathcal{J}$ the sheaf of flat sections along the polarization, we can then define the quantization as $\mathcal{Q}(M)=\bigoplus_{k>0} H^{k}(M, \mathcal{J})$. Then quantization is given by precisely the following theorem of Sniatycki [66]:

Theorem 2.5 (Sniatycki). If the leaf space of the polarization $B^{n}$ is a Hausdorff manifold and the natural projection $\pi: M^{2 n} \rightarrow B^{n}$ is a fibration with compact fibres, then all the cohomology groups vanish except for degree half of the dimension of the manifold. Furthermore, $\mathcal{Q}\left(M^{2 n}\right)=H^{n}\left(M^{2 n}, \mathcal{J}\right)$, and the dimension of $H^{n}\left(M^{2 n}, \mathcal{J}\right)$ is the number of Bohr-Sommerfeld leaves.

2.2. Singular action-angle coordinates. In view of the "symplectic" uniqueness for the problem of classification in the case of regular compact orbits, we want to try to extend this symplectic uniqueness also to fibrations which are not regular but admit some "normal forms".

What can be said about the corresponding classification problem for symplectic germs if the completely integrable systems has singularities?

This question is quite natural because singularities are present in many wellknown examples of integrable systems. In fact, if the completely integrable system is defined on a compact manifold then the singularities cannot be avoided.

If we forget for a while about the symplectic structure now and think about the singularities that the moment map $\mathbb{F}$ can have. A good starting point is to consider singularities which are non-degenerate (Morse-Bott) because for these singularities such "normal forms" for the fibration do exist. We may wish to try to classify symplectic germs in a neighbhourhood of a singular compact orbit as well. But in this case because the moment map has singularities and there will be singular orbits, this is why the foliation will not longer be Lagrangian but isotropic. Indeed most of the leaves will be Lagrangian. This is why we talk about the "generically" Lagrangian foliation. In this case we get a uniqueness of the symplectic structure for each linear symplectic model and the result that we prove can be seen as a "singular" Liouville-Mineur-Arnold theorem.

In [49] we proved that the uniqueness result for symplectic germs for which the foliation determined by a completely integrable system is generically Lagrangian holds when $L$ is a singular compact orbit.

In the singular case, the problem can be posed at three different levels in the neighbourhood of an orbit, levelset or globally. We will only address the first point of view here.

In this paper we will sketch a different proof of Eliasson's normal form [21], [20] in the context of linearization of associated foliation (not moment map) for general Williamson type. The proof that we will discuss here is essentially a slightly more sophisticated proof that the one in the author's thesis [49].

Observe that this normal form theorem can be seen as a symplectic linearization result which ensures that the initial completely integrable system can be taken to the linear system and that the symplectic form can be taken to the standard 
one. As a byproduct, we obtain a multiple differentiable linearization result for $n$ commuting vector fields with singularities of non-degenerate type.

The symplectic linearization in a neighbourhood of a singular non-degenerate orbit $L$ with $\operatorname{dim} L>0$ is due to Ito in the analytic case [33]. Partial results in that direction (with $\operatorname{dim} L=1$ in a manifold of dimension 4) where obtained by Currás-Bosch and the author in [10] and independently by Colin de Verdière and $\mathrm{San} \mathrm{Vu}$ Ngoc in [9]. The final result in any dimension was obtained by Nguyen Tien Zung and the author in [58]. In [58] it is also included a $G$-equivariant version of the symplectic linearization.

The problem of topological classification of integrable Hamiltonian systems began with Fomenko [22] in some particular cases. Nguyen Tien Zung [77] studied the general case for the semi-local problem for non-degenerate singularities. It turns out that from a topological point of view we have a product-like description of the singularities in terms of the Williamson type. Nguyen Tien Zung also proved in [77] the existence of partial action-angle coordinates. The symplectic classification in the semi-local case for non-elliptic singularities has been studied in the hyperbolic case by Dufour, Molino and Toulet in [17]. The focus-focus case has been studied recently by San Vu Ngoc in [71]. In the hyperbolic and focus-focus case there are more invariants attached to the singularity. The symplectic germ in the hyperbolic case is determined by the jet of a function depending on a variable and in the focus-focus case is determined by the jet of a function in two variables. The singular global case has been studied by Nguyen Tien Zung in the paper [79] where the notion of Duistermaat-Chern class and monodromy (introduced by Duistermaat for regular foliations) is extended in order to include the singularities into the picture.

The condition of non-degeneracy is always present in the works cited above. There are also some contributions for degenerate singularities in the world of integrable systems. A recent contribution in that direction is contained in the paper [7] by Colin de Verdière. In that paper, among other things, the problem of classification of germs of singular Lagrangian manifolds is posed for more general singularities with a special emphasis on quasi-homogeneous singularities. For instance in this paper an explicit classification is obtained in the case of the cusp.

The singular analogue of Liouville-Mineur-Arnold theorem was considered by Eliasson in his thesis [20]. He constructed singular action coordinates for a special type of non-degenerate singularities. This is a major breakthrough which uses a clever combination of analysis and Moser's path method. However, there were some inaccuracies in some statements which lead to some confusion in the literature. We will try to clarify those here.

The singular achievements formerly specified often have a semiclassical version. Their semiclassical counterpart has been obtained by Colin de Verdière and San Vu Ngoc in $[9,7]$.

2.2.1. Normal forms for non-degenerate singularities in a neigbourhood of a point. The singularity of the orbit can be described in terms of the singularity of the functions $f_{i}$.

Assume first that the rank of the differential at the point is zero, since the functions $f_{i}$ are in involution with respect to the Poisson bracket, the quadratic parts of the functions $f_{i}$ commute defining in this way an abelian subalgebra of 
$Q(2 n, \mathbb{R})$ (the set of quadratic forms on $2 n$-variables). In the case the singularity of the functions $f_{i}$ is of Morse type this subalgebra is indeed a Cartan subalgebra. We call these singularities of non-degenerate type.

The problem of classification of singularities for the quadratic parts of the functions $f_{i}$ can be therefore converted into the problem of classification of Cartan subalgebras of $Q(2 n, \mathbb{R})$. The singularities for the quadratic parts are well-understood thanks to a result of Williamson [76] where Cartan subalgebras of $Q(2 n, \mathbb{R})$ are classified. Let us recall its precise statement,

Theorem 2.6. (Williamson) For any Cartan subalgebra $\mathcal{C}$ of $Q(2 n, \mathbb{R})$ there is a symplectic system of coordinates $\left(x_{1}, \ldots, x_{n}, y_{1}, \ldots, y_{n}\right)$ in $\mathbb{R}^{2 n}$ and a basis $f_{1}, \ldots, f_{n}$ of $\mathcal{C}$ such that each $f_{i}$ is one of the following:

$$
\begin{aligned}
& f_{i}=x_{i}^{2}+y_{i}^{2} \quad \text { for } 1 \leq i \leq k_{e} \text {, } \\
& f_{i}=x_{i} y_{i} \quad \text { for } k_{e}+1 \leq i \leq k_{e}+k_{h} \text {, } \\
& \left\{\begin{array}{l}
f_{i}=x_{i} y_{i+1}-x_{i+1} y_{i}, \quad \text { (focus-focus pair) } \\
f_{i+1}=x_{i} y_{i}+x_{i+1} y_{i+1} \quad \text { for } i=k_{e}+k_{h}+2 j-1,1 \leq j \leq k_{f}
\end{array}\right.
\end{aligned}
$$

The linear system given by the quadratic parts of the $f_{i}$ is called the linear model for a singularity. We may attach a triple of natural numbers $\left(k_{e}, k_{h}, k_{f}\right)$ to a nondegenerate singularity $p$ of $F$, where $k_{e}$ stand for the number of elliptic components in the linear model, $k_{h}$ and $k_{f}$ the number of hyperbolic and focus-focus components in the linear model respectively. By virtue of Williamson theorem this triple is an invariant of the linear system. That is why this triple is often called the Williamson type of the singularity. The linear model for fixed points is given by Williamson's theorem stated above. Now a natural question arises:

Can we linearize the completely integrable system symplectically in a neighbourhood of a point $p$ ?

We can reformulate the question as follows,

\section{Problem $1^{2}$}

Consider a foliation $\mathcal{F}$ defined by a completely integrable system defined in a neighbourhood of a non-degenerate singular 0-dimensional orbit of $\mathcal{F}$ of Williamson type $\left(k_{e}, k_{h}, k_{f}\right)$. Assume that we are given two symplectic forms $\omega_{1}$ and $\omega_{2}$ for which the foliation $\mathcal{F}$ is Lagrangian. Does there exist a local diffeomorphism fixing $p$ preserving the foliation $\mathcal{F}$ and taking $\omega_{1}$ to $\omega_{2}$ ?

\footnotetext{
${ }^{2}$ This problem of symplectic linearization is closely related to another problem in the spirit of Morse lemma which was solved succesfully by Vey for analytic systems [68] and by Vey and Colin de Verdière [8] for smooth systems. Problem 2: Given a function $f: \mathbb{R}^{n} \longrightarrow \mathbb{R}$ with a non-degenerate singularity at the origin and let $\omega$ be a volume form on $\mathbb{R}^{n}$ and let $Q$ be its quadratic part at the origin. Does there exist a diffeomorphism $\phi:\left(R^{n}, 0\right) \longrightarrow\left(R^{n}, 0\right)$ such that $\phi^{*}(f)=Q$ and such that $\omega$ is taken to the volume form $\omega_{0}=d x_{1} \wedge \cdots \wedge d x_{n}$ ? In [8] Colin de Verdière and Vey prove that there exists a smooth function $\chi$ such that $\phi^{*}(\omega)=\chi(Q) \cdot \omega_{0}$.In that paper it is also proved that the function $\chi$ is characteristic of the pair $(f, \omega)$ if $Q$ is definite, otherwise only the jet is characteristic for the pair. As a consequence of this result we obtain normal forms for foliations defined by the levelsets of $f$ because we can find a foliation-preserving diffeomorphism sending the volume form $\chi(Q) \cdot \omega_{0}$ to the volume form $\omega_{0}$. Notice as well that this result provides an affirmative answer to Problem 1 in the case $n=2$ because a volume form on a 2-dimensional manifold is a symplectic form and the Lagrangian condition for a curve is automatic in that dimension.
} 
The affirmative answer to Problem 1 in any dimension was provided in the elliptic case by Eliasson in [21]. As a matter of fact the proof provided by Eliasson seems complete just in the case the singularity is completely elliptic (of Williamson type $\left.\left(k_{e}, 0,0\right)\right) .^{3}$

In this paragraph we state the analogue of Eliasson's theorem for general singularities, using foliations. Other statements using moment maps and additional hypotheses on the bifurcation diagrams have been provided by [45] and [6].

Assume that $\mathcal{F}$ is a linear foliation on $M^{2 n}$ with a rank 0 singularity at the origin $p$. Assume that the Williamson type of the singularity is $\left(k_{e}, k_{h}, k_{f}\right)$. The linear model for the foliation is then generated by the following vector fields,

$$
\begin{aligned}
& X_{i}=-y_{i} \frac{\partial}{\partial x_{i}}+x_{i} \frac{\partial}{\partial y_{i}} \text { for } 1 \leq i \leq k_{e}, \\
& X_{i}=y_{i} \frac{\partial}{\partial y_{i}}-x_{i} \frac{\partial}{\partial x_{i}} \text { for } k_{e}+1 \leq i \leq k_{e}+k_{h}, \\
& X_{i}=x_{i} \frac{\partial}{\partial x_{i+1}}-y_{i+1} \frac{\partial}{\partial y_{i}}-x_{i+1} \frac{\partial}{\partial x_{i}}+y_{i} \frac{\partial}{\partial y_{i+1}} \text { and } \\
& X_{i+1}=-x_{i} \frac{\partial}{\partial x_{i}}+y_{i} \frac{\partial}{\partial y_{i}}-x_{i+1} \frac{\partial}{\partial x_{i+1}}+y_{i+1} \frac{\partial}{\partial y_{i+1}} \text { for } i=k_{e}+k_{h}+2 j-1,1 \leq j \leq k_{f}
\end{aligned}
$$

We can prove the following symplectic linearization result [49],

Theorem 2.7. Let $\omega$ be a symplectic form defined in a neighbourhood of the origin for which $\mathcal{F}$ is Lagrangian, then there exists a local diffeomorphism $\phi:(U, p) \longrightarrow$ $(\phi(U), p)$ such that $\phi$ preserves the foliation and $\phi^{*}\left(\sum_{i} d x_{i} \wedge d y_{i}\right)=\omega$, being $x_{i}, y_{i}$ local coordinates on $(\phi(U), p)$.

Remark 2.8. In the case the singularities are completely elliptic, this is equivalent to Eliasson's theorem [21].

2.2.2. Normal forms for non-degenerate singularities in a neigbourhood of an orbit. Normal forms for higher rank have been obtained jointly with Nguyen Tien Zung [58].

These normal form results can be seen as a symplectic Morse-Bott theorem for integrable systems. The singular fibers can have non-compact symmetry group associated to it. This is why in order to obtain a complete proof of the symplectic equivalence problem, we need to consider the equivariant version of the statements which we will see in the next subsection.

In the case the rank $k$ of the differential of the moment map is greater than 0 , we may reduce the problem of classification to the fixed point case via a reduction by a Hamiltonian action of $\mathbb{T}^{k}$ which preserves the foliation.

In [77] it is proved that,

Theorem 2.9. Let $U(L)$ be a neighbourhood of a nondegenerate singular compact orbit of an integrable system with $n$ degrees of freedom. Assume the corank of the orbit is $n-k=k_{e}+k_{h}+2 k_{f}$. Let $\mathcal{F}$ be the singular Lagrangian foliation defined by the integrable system. Then there exists a normal finite covering $\widetilde{U(L)}$ of $U(L)$ such that the foliation can be lifted to $\widetilde{\mathcal{F}}$ and a free Hamiltonian action of the torus $\mathbb{T}^{k}$ in the covering $\widetilde{U(L)}$ which preserves the moment map.

\footnotetext{
${ }^{3}$ What is not true in general is that the moment map has component functions which are, in turn, functions of the basis of the Cartan subalgebra. This fails essentially in the case the system has hyperbolic components.
} 
Now we can use this normal finite covering and introduce the linear model associated to the orbit $L$. Later, we will see that the invariants associated to the linear model are the Williamson type of the orbit and a twisting group $\Gamma$ attached to it.

Denote by $\left(p_{1}, \ldots, p_{k}\right)$ a linear coordinate system of a small ball $D^{k}$ of dimension $k,\left(\theta_{1}, \ldots, \theta_{k}\right)$ is a standard periodic coordinate system of the torus $\mathbb{T}^{k}$, and $\left(x_{1}, y_{1}, \ldots, x_{n-k}, y_{n-k}\right)$ a linear coordinate system of a small ball $D^{2(n-k)}$ of dimension $2(n-k)$. Now we consider the manifold

$$
V=D^{k} \times \mathbb{T}^{k} \times D^{2(n-k)}
$$

with the standard symplectic form $\sum d p_{i} \wedge d \theta_{i}+\sum d x_{j} \wedge d y_{j}$, and the following moment map:

$$
F=\left(p_{1}, \ldots, p_{k}, f_{1}, \ldots, f_{n-k}\right): V \rightarrow \mathbb{R}^{n}
$$

where

$$
\begin{aligned}
& f_{i}=x_{i}^{2}+y_{i}^{2} \text { for } 1 \leq i \leq k_{e}, \\
& f_{i}=x_{i} y_{i} \text { for } k_{e}+1 \leq i \leq k_{e}+k_{h}, \\
& f_{i}=x_{i} y_{i+1}-x_{i+1} y_{i} \text { and } \\
& f_{i+1}=x_{i} y_{i}+x_{i+1} y_{i+1} \text { for } i=k_{e}+k_{h}+2 j-1,1 \leq j \leq k_{f}
\end{aligned}
$$

The linearized foliation in the covering is the foliation determined by the above moment map. After performing a linear change of coordinates in such a way that the hyperbolic functions can be written as $f_{i}=x_{i}^{2}-y_{i}^{2}$, the following vector fields form a basis of $\chi$,

$$
\begin{aligned}
& Y_{i}=\frac{\partial}{\partial \theta_{i}} \text { for } 1 \leq i \leq k, \\
& X_{i}=-y_{i} \frac{\partial}{\partial x_{i}}+x_{i} \frac{\partial}{\partial y_{i}} \text { for } 1 \leq i \leq k_{e}, \\
& X_{i}=y_{i} \frac{\partial}{\partial x_{i}}+x_{i} \frac{\partial}{\partial y_{i}} \text { for } k_{e}+1 \leq i \leq k_{e}+k_{h}, \\
& X_{i}=x_{i} \frac{\partial}{\partial x_{i+1}}-y_{i+1} \frac{\partial}{\partial y_{i}}-x_{i+1} \frac{\partial}{\partial x_{i}}+y_{i} \frac{\partial}{\partial y_{i+1}} \text { and } \\
& X_{i+1}=-x_{i} \frac{\partial}{\partial x_{i}}+y_{i} \frac{\partial}{\partial y_{i}}-x_{i+1} \frac{\partial}{\partial x_{i+1}}+y_{i+1} \frac{\partial}{\partial y_{i+1}} \text { for } i=k_{e}+k_{h}+2 j-1,1 \leq j \leq k_{f}
\end{aligned}
$$

In order to prove equivalence in a neighbourhood of an orbit we need to first consider additional symmetries corresponding to the deck-transformations. We prove it for general symplectic actions preserving the system.

2.2.3. Additional symmetries. We assume also that the group acts symplectically and preserves the moment map which is underlying in the foliation.

We end up proving the equivariant version of the symplectic uniqueness result in a neighbourhood of a singular compact orbit. This result is contained in [49] and [58].

We are going to introduce the notion of linear action on the linear model associated to the orbit $L$ for a given symplectic action preserving the system. Later, we will see that the invariants associated to the linear model are the Williamson type of the orbit and a twisting group $\Gamma$ attached to it.

Let $\Gamma$ be a group with a symplectic action $\rho(\Gamma)$ on $V$, which preserves the moment map $\mathbf{F}$. We will say that the action of $\Gamma$ on $V$ is linear if it satisfies the following property:

$\Gamma$ acts on the product $V=D^{k} \times \mathbb{T}^{k} \times D^{2(n-k)}$ componentwise; the action of $\Gamma$ on $D^{k}$ is trivial, its action on $\mathbb{T}^{k}$ is by translations (with respect to the coordinate 
system $\left.\left(\theta_{1}, \ldots, \theta_{k}\right)\right)$, and its action on $D^{2(n-k)}$ is linear with respect to the coordinate system $\left(x_{1}, y_{1}, \ldots, x_{n-k}, y_{n-k}\right)$.

Suppose now that $\Gamma$ is a finite group with a free symplectic action $\rho(\Gamma)$ on $V$, which preserves the moment map and which is linear. Then we can form the quotient symplectic manifold $V / \Gamma$, with an integrable system on it given by the induced moment map as above:

$$
\mathbf{F}=\left(p_{1}, \ldots, p_{k}, f_{k+1}, \ldots, f_{n}\right): V / \Gamma \rightarrow \mathbb{R}^{n}
$$

The set $\left\{p_{i}=x_{i}=y_{i}=0\right\} \subset V / \Gamma$ is a compact orbit of Williamson type $\left(k_{e}, k_{f}, k_{h}\right)$ of the above system. We will call the above system on $V / \Gamma$, together with its associated singular Lagrangian foliation, the linear system (or linear model) of Williamson type $\left(k_{e}, k_{f}, k_{h}\right)$ and twisting group $\Gamma$ (or more precisely, twisting action $\rho(\Gamma))$. We will also say that it is a direct model if $\Gamma$ is trivial, and a twisted model if $\Gamma$ is nontrivial.

A symplectic action of a compact group $G$ on $V / \Gamma$ which preserves the moment map $\left(p_{1}, \ldots, p_{k}, f_{k+1}, \ldots, f_{n}\right)$ will be called linear if it comes from a linear symplectic action of $G$ on $V$ which commutes with the action of $\Gamma$. In our case, let $\mathcal{G}^{\prime}$ denote the group of linear symplectic maps which preserve the moment map then this group is abelian and therefore this last condition is always satisfied.

In [49] and [58] we prove the following:

Theorem 2.10 (Miranda-Zung). Consider $\mathcal{F}$ the foliation defined by a completely integrable system and consider $L$, a compact orbit of dimension $k$ and of Williamson type $\left(k_{e}, k_{h}, k_{f}\right)$. Let $\omega$ be a symplectic for which the foliation $\mathcal{F}$ is Lagrangian. Then there exists a finite group $\Gamma$ and a diffeomorphism taking the foliation to the linear foliation on $V / \Gamma$ given by the linear model above, and taking $\omega$ to $\omega_{0}$, which sends $L$ to the torus $\left\{p_{i}=x_{i}=y_{i}=0\right\}$. The smooth symplectomorphism $\phi$ can be chosen so that via $\phi$, the system-preserving action of the compact group $G$ near $L$ becomes a linear system-preserving action of $G$ on $V / \Gamma$. If the moment map $\mathbf{F}$ is real analytic and the action of $G$ near $L$ is analytic, then the symplectomorphism $\phi$ can also be chosen to be real analytic. If the system depends smoothly (resp., analytically) on a local parameter (i.e. we have a local family of systems), then $\phi$ can also be chosen to depend smoothly (resp., analytically) on that parameter.

Remark 2.11. A nice consequence is the abelianity of the connected component of the identity of the group of symplectomorphisms preserving the moment map.

Remark 2.12. This theorem has interesting applications to geometric quantization of singular real polarizations (see [53] and [30]).

2.2.4. Some words about the proof of the singular counterpart to Liouville-MineurArnold. In this section we outline a proof for equivariant symplectic equivalence with the linear models for non-degenerate orbits.

These results are contained in [49] and [58]. We distinguish in the proof the rank 0 case from the general rank case. The proof that we outline here for the rank 0 case is an improved version of the proof contained in the author's thesis. This complete proof which is based on Mather's principle "infinitesimal rigidity implies rigidity "will be included in [54].

Reduction from the general rank case to the 0-rank case (theorem 2.7) 
In [58] and [49] we use the following strategy: There is a Hamiltonian $\mathbb{T}^{k}$ action $\rho$ tangent to the singular Lagrangian foliation. Compose now with the inverse action in such a way that now points in the singular orbit become fixed points, take a transversal to the orbit and use the 0-rank case (theorem 2.7). Pick a point on the singular orbit. Now in this neighbourhood and because the point is fixed, we can apply theorem 0-rank case to linearize the foliation. Use now the initial Hamiltonian $\mathbb{T}^{k}$ action $\rho$ to drag this linearization result along the orbit.

To conclude the case of additional symmetries, we just need the following result contained in [58] (proposition 3.6), Denote by $\rho$ the action of a compact group $G$ on $\left(\mathbb{R}^{2 n}, \sum_{i=1}^{n} d x_{i} \wedge d y_{i}, \mathbf{h}\right)$ where $\mathbf{h}$ is the linear moment map of the model at a fixed point.

Proposition 2.13. Then there exist a local symplectic variable transformation of $\mathbb{R}^{2 n}$ which preserves the system $\left(\mathbb{R}^{2 n}, \sum_{i=1}^{n} d x_{i} \wedge d y_{i}, \mathbf{h}\right)$ and under which the action of $G$ becomes linear.

The combination of theorem 2.7 with this proposition gives the proof of theorem 2.10 .

Proof of the rank 0 case without additional symmetries: Theorem 2.7

Here we outline the proof of Theorem 2.7 which is included in [49]. We present an improved version of this proof which uses infinitesimal rigidity and symplectic orthogonal decomposition to conclude. Details about this proof will be included in [54].

Steps of the proof:

(1) Step 1- Differentiable linearization. We prove that the foliation can be assumed to be the one of the linear model. It is important to stress here that there is no manipulation of the symplectic form at this stage.

(2) Step 2- Analytical tools. In order to work with the symplectic form and its coeffients we need to introduce some analytical tools that concern special decomposition of functions in basic and non-basic parts.

(3) Step 3- Infinitesimal rigidity. We prove a singular Poincaré lemma. This was proved with San Vu Ngoc and the author in [57]. This singular Poincaré lemma entails infinitesimal rigidity.

(4) Step 4- Infinitesimal rigidity implies rigidity. We use the singular Poincaré lemma to solve a cohomological equation related to the symplectic structure in a neighbourhood of the singular orbit. The solution of this cohomological equation allows to deform the symplectic structure in a foliation preserving way via Moser's path method.

We can use these Moser type arguments to attain a symplectically orthogonal decomposition of our system in 2 dimensional and 4-dimensional blocks. The 2-dimension blocks correspond to elliptic and hyperbolic singularities and the 4-dimensional blocks carry focus-focus singularities.

By means of this symplectically orthogonal decomposition we reduce our problem of symplectic linearization to the study of symplectic linearization in dimension 2 for elliptic and hyperbolic singularities and in dimension 4 for focus-focus singularities. It turns out that the proof of these cases is already contained in the literature ([21] for the elliptic case, [8] for the hyperbolic case and [71], [72], [11] and [20] for the focus-focus case). 
Remark 2.14. It is very important to stress out that the proof for the completely elliptic case was completed before by Eliasson in [21]. In [20] one can find an statement for the general case (including the focus-focus and hyperbolic case). This statement seems is not accurate in the case there are hyperbolic singularities. The main problem is that we cannot ensure that the moment map will be a function of the basis of the Williamson basis. This inaccuracy has propagated somehow in the literature of integrable systems and had already been detected by the author [49] and other authors. A different approach and hypothesis are contained in the works of Matveev and Bolsinov [6] and [45]).

We will know expand a little further Steps 2 and 3 of the proof.

\section{Step 2 Analytical tools in the proof of Theorem 2.7}

We start with two special decomposition for functions. Let $g$ be a smooth function if $X$ is a smooth vector field on a manifold $M$ and $p \in M$ such that $X(p) \neq 0$, then it is a well-known result that $g$ admits a local smooth decomposition of the following type:

$$
g=g_{1}+X\left(g_{2}\right) \quad, \quad X\left(g_{1}\right)=0
$$

In order to do that just take local coordinates $\left(x_{1}, \ldots, x_{n}\right)$ centered at a point $p$ such that $X=\frac{\partial}{\partial x_{1}}$ and apply the classical integration trick. That is, if we consider the smooth function $g_{1}\left(x_{1}, \ldots, x_{n}\right)=g\left(0, x_{2}, \ldots, x_{n}\right)$ and the smooth function

$$
g_{2}=\int_{0}^{1} g\left(t x_{1}, \ldots, x_{n}\right) d t
$$

we obtain the desired decomposition 2.7.

Now the question arises: Can we obtain similar local decomposition for singular vector fields?

We can prove that similar decompositions can be obtained for the following vector fields $X=x_{1} \frac{\partial}{\partial x_{2}}-x_{2} \frac{\partial}{\partial x_{1}}$ or $Y=-x_{1} \frac{\partial}{\partial x_{1}}+x_{2} \frac{\partial}{\partial x_{2}}$.

These results are contained in Chapter 2 of the authors' thesis [49]. We give here statements and proofs which are included there and give a flavour of the analysis involved in the proofs. In [57] a combined results of these decomposition with additional crossed derivatives properties is included.

This first proposition is proved by Eliasson in [21] and [20] in any dimension whereas a proof for the second proposition is proved by Eliasson when $n=2$ in [20]. Let us point out that when the manifold is $M=\mathbb{R}^{2}$ a proof of this decomposition had been formerly given by Guillemin and Schaeffer [26] and by Colin de Verdière and Vey [8]. This generalization to any dimension seems to be new in the nonelliptic case. In any case the techniques used here are fairly inspired in those of the paper of Colin de Verdière and Vey.

Proposition 2.15. Let $M$ be a differentiable manifold and let $g$ be a germ of smooth function in a neighbourhood of a point $p$. Consider $X$ a vector field which in local coordinates can be written as $X=x_{1} \frac{\partial}{\partial x_{2}}-x_{2} \frac{\partial}{\partial x_{1}}$ then there exist differentiable functions $g_{1}$ and $g_{2}$ such that:

$$
g=g_{1}\left(x_{1}^{2}+x_{2}^{2}, x_{3} \ldots, x_{n}\right)+X\left(g_{2}\right)
$$

For hyperbolic singularities we obtain the following 
Proposition 2.16. Let $M$ be a differentiable manifold and let $g$ be a germ of smooth function in a neighbourhood of a point $p$. Consider $X$ a vector field which in local coordinates can be written as $Y=-x_{1} \frac{\partial}{\partial x_{1}}+x_{2} \frac{\partial}{\partial x_{2}}$ then there exist differentiable functions $g_{1}$ and $g_{2}$ such that

$$
g=g_{1}\left(x_{1} x_{2}, x_{3} \ldots, x_{n}\right)+Y\left(g_{2}\right)
$$

Before we will need some lemmas concerning the smooth resolution of the equation $Y(f)=g$ for a given smooth $g$.

Lemma 2.17. Let $g$ be a smooth function, the equation $Y(f)=g$ admits a formal solution along the subspace $S=\left\{\left(0,0, x_{3}, \ldots, x_{n}\right)\right\}$ if and only if

$$
\frac{\partial^{2 k} g}{\partial x_{1}^{k} \partial x_{2}^{k}}\left(0,0, x_{3}, \ldots, x_{n}\right)=0 .
$$

Proof. Let us construct a solution considering the $\left(x_{1}, x_{2}\right)$-jets. That is, assume the $\left(x_{1}, x_{2}\right)$-jet of $f$ along $S=\left\{\left(0,0, x_{3}, \ldots, x_{n}\right)\right\}$ is $\sum_{i j} f_{i j} x_{1}^{i} x_{2}^{j}$, the coefficients $f_{i j}$ being functions in the variables $\left(x_{3}, \ldots, x_{n}\right)$. Denote by $\sum_{i j} g_{i j} x_{1}^{i} x_{2}^{j}$ the $\left(x_{1}, x_{2}\right)$ jet of $g$ along $S=\left\{\left(0,0, x_{3}, \ldots, x_{n}\right)\right\}$. Then the condition $X(f)=g$ implies the following conditions for the coefficient functions

$$
(-i+j) f_{i j}=g_{i j} \quad, \quad \forall i, j
$$

Particularizing $i=j$ in this equation we obtain $g_{i i}=0$; so in order to have a solution by jets of the equation $Y(f)=g$, the terms $\frac{\partial^{2 k} g}{\partial x_{1}^{k} \partial x_{2}^{k}}\left(0,0, x_{3}, \ldots, x_{n}\right)$ have to vanish necessarily.

On the other hand if $i \neq j$ from the above relation, the following relation is met $f_{i j}=\frac{g_{i j}}{-i+j}$. Therefore, if the condition $\frac{\partial^{2 k} g}{\partial x_{1}^{k} \partial x_{2}^{k}}\left(0,0, x_{3}, \ldots, x_{n}\right)=0$ is fulfilled this gives a solution by jets to the equation $Y(f)=g$.

According to Borel's theorem there exists a smooth function $\widehat{f}$ with the $\left(x_{1}, x_{2}\right)$ jets previously found. It remains to solve this equation for functions for which

$$
\frac{\partial^{i+j} g}{\partial x_{1}^{i} \partial x_{2}^{j}}\left(0,0, x_{3}, \ldots, x_{n}\right)=0 .
$$

We will refer to this functions as $\left(x_{1}, x_{2}\right)$-flat functions along the subspace $S=$ $\left\{\left(0,0, x_{3}, \ldots, x_{n}\right)\right\}$.

Lemma 2.18. Let $g$ be a $\left(x_{1}, x_{2}\right)$-flat function along the subspace $S=\left\{\left(0,0, x_{3}, \ldots, x_{n}\right)\right\}$ then there exists a smooth function $f$ for which $Y(f)=g$.

Proof. Consider the function,

$$
T\left(x_{1}, \ldots, x_{n}\right)= \begin{cases}\frac{1}{2} \ln \frac{x_{1}}{x_{2}} & x_{1} x_{2}>0 \\ \frac{1}{2} \ln \frac{-x_{1}}{x_{2}} & x_{1} x_{2}<0\end{cases}
$$

Denote by $\phi_{t}\left(x_{1}, \ldots, x_{n}\right)$ the flow of the vector field $Y$, being $Y=-x_{1} \frac{\partial}{\partial x_{1}}+$ $x_{2} \frac{\partial}{\partial x_{2}}$. Observe that $\phi_{t}\left(x_{1}, \ldots, x_{n}\right)=\left(e^{-t} x_{1}, e^{t} y_{1}, \ldots, x_{n}\right)$.

Now we define 


$$
f\left(x_{1}, \ldots, x_{n}\right)=-\int_{0}^{T\left(x_{1}, \ldots, x_{n}\right)} g\left(\phi_{t}\left(x_{1}, \ldots, x_{n}\right)\right) d t .
$$

This function is defined outside the set $\Omega=\Omega_{1} \cup \Omega_{2}$ being $\Omega_{1}=\left\{\left(x_{1}, \ldots, x_{n}\right), x_{1}=\right.$ $0\}$ and $\Omega_{2}=\left\{\left(x_{1}, \ldots, x_{n}\right), x_{2}=0\right\}$. We now prove that $f$ admits a smooth continuation in the whole neighbourhood considered and that it is a solution to our problem.

Formally differentiating under the integral sign, the computation of the first derivatives reads,

(1) If $i=1,2$

$$
\frac{\partial}{\partial x_{i}} f=-g\left(\phi_{T\left(x_{1}, x_{2}, \ldots, x_{n}\right)}\right) \frac{\partial}{\partial x_{i}} T-\int_{0}^{T\left(x_{1}, \ldots, x_{n}\right)} \frac{\partial}{\partial x_{i}} g\left(\phi_{t}\left(x_{1}, \ldots, x_{n}\right)\right) d t
$$

(2) When $i \neq 1$ and $i \neq 2$,

$$
\frac{\partial}{\partial x_{i}} f=-\int_{0}^{T\left(x_{1}, \ldots, x_{n}\right)} \frac{\partial}{\partial x_{i}} g\left(\phi_{t}\left(x_{1}, \ldots, x_{n}\right)\right) d t
$$

Observe that the set $S$ equals $S=\Omega_{1} \cap \Omega_{2}$. Observe that $f$ is smooth outside the set $\Omega=\Omega_{1} \cup \Omega_{2}$.

The first term in 2.9 is smooth outside the set $\Omega=\Omega_{1} \cup \Omega_{2}$. And observe that if $p$ lies in $\Omega$ then from the definition of $T$, the point $\phi_{T}(p)$ lies in $S$. On the other hand, the function $g$ is flat along the subspace $S$. Thus the first term in $2.9-g\left(\phi_{T\left(x_{1}, x_{2}, \ldots, x_{n}\right)}\right) \frac{\partial}{\partial x_{i}} T$ is smooth in the whole neighbourhood of the origin considered.

As for the second term, we could reproduce word by word the proof supplied by Eliasson in [20] in the two dimensional case. The proof can be adapted because the function $g$ is flat along $S$. In fact, it is just the parametric version of Eliasson's result. In the same way, Eliasson's proof yields that the integral 2.10 is a smooth function.

The same arguments applied to the successive derivatives prove that $f$ admits a $\mathcal{C}^{\infty}$ continuation.

Now let us check that this is a solution to the equation.

First,

$$
f\left(\phi_{s}\left(x_{1}, \ldots, x_{n}\right)\right)=-\int_{0}^{T\left(\phi_{s}\left(x_{1}, \ldots, x_{n}\right)\right)} g\left(\phi_{s}\left(\phi_{t}\left(x_{1}, \ldots, x_{n}\right)\right)\right) d t
$$

The relations $\ln \frac{e^{-s} x_{1}}{e^{s} x_{2}}=\ln \frac{x_{1}}{x_{2}}-2 s$ when $x_{1} x_{2} \geq 0$ and $\ln \frac{-e^{-s} x_{1}}{e^{s} x_{2}}=\ln \frac{-x_{1}}{x_{2}}-2 s$ when $x_{1} x_{2} \leq 0$ imply $T\left(\phi_{s}\left(x_{1}, \ldots, x_{n}\right)\right)=T\left(x_{1}, \ldots, x_{n}\right)-s$. On the other hand, since $\phi_{s}$ is a one-parameter subgroup. Equation 2.11 can be written as,

$$
f\left(\phi_{s}\left(x_{1}, \ldots, x_{n}\right)\right)=-\int_{0}^{T\left(x_{1}, \ldots, x_{n}\right)-s} g\left(\phi_{t+s}\left(x_{1}, \ldots, x_{n}\right)\right) d t
$$


Now we perform the change of variable $\bar{t}=t+s$ and this equation reads,

$$
f\left(\phi_{s}\left(x_{1}, \ldots, x_{n}\right)\right)=-\int_{s}^{T\left(x_{1}, \ldots, x_{n}\right)} g\left(\phi_{\bar{t}}\left(x_{1}, \ldots, x_{n}\right)\right) d \bar{t}
$$

Now after differentiating in $s$ this equation yields,

$$
\frac{d f\left(\phi_{s}\left(x_{1}, \ldots, x_{n}\right)\right)}{d s}=g\left(\phi_{s}\left(x_{1}, \ldots, x_{n}\right)\right)
$$

Finally, put $s=0$ to obtain $Y(f)=g$ as we wanted. This ends the proof of the lemma.

Let us go back to the proof of proposition 2.16. Given a differentiable function $g$, we want to find smooth functions $g_{1}$ and $g_{2}$ such that

$$
g=g_{1}\left(x_{1} x_{2}, x_{3} \ldots, x_{n}\right)+Y\left(g_{2}\right) .
$$

The strategy for finding this decomposition is to find a solution by $\left(x_{1}, x_{2}\right)$-jets and then apply the second lemma to gather all the remaining $\left(x_{1}, x_{2}\right)$-flat terms as $Y(f)$ for a certain smooth $f$.

So let $\sum_{i j} g_{i j} x_{1}^{i} x_{2}^{j}$ be the $\left(x_{1}, x_{2}\right)$-Taylor expand for $g$ at a point $\left(0,0, x_{3}, \ldots, x_{n}\right)$ lying in the subspace $S=\left\{\left(0,0, x_{3}, \ldots, x_{n}\right)\right\}$.

Now we split this Taylor expand in two. The first one, $\sum_{i i} g_{i j} x_{1}^{i} x_{2}^{i}$, and the second one $\sum_{i \neq j} g_{i j} x_{1}^{i} x_{2}^{j}$. Denote by $\widehat{r}_{1}$ and $\widehat{r}_{2}$ two smooth functions with the previous jets. Then we can assert that

$$
\widehat{r}_{1}=g_{1}\left(x_{1} x_{2}, x_{3}, \ldots, x_{n}\right)+\phi\left(x_{1}, \ldots, x_{n}\right),
$$

being $\phi\left(x_{1}, \ldots, x_{n}\right)$ a $\left(x_{1}, x_{2}\right)$-flat function along $S=\left\{\left(0,0, x_{3}, \ldots, x_{n}\right)\right\}$. Further, using the two above lemmas $(2.17,2.18)$, the function $\widehat{r}_{2}$ can be written as $\widehat{r}_{2}=Y\left(R_{2}\right)$. Now since $\phi$ is $\left(x_{1}, y_{1}\right)$-flat, according to lemma 2.18 we can write $\phi\left(x_{1}, \ldots, x_{n}\right)=Y(R)$. Finally define $g_{2}=R_{2}+R$ and $g_{1}$ and $g_{2}$ satisfy the decomposition sought $g=g_{1}\left(x_{1} x_{2}, x_{3} \ldots, x_{n}\right)+Y\left(g_{2}\right)$. And this completes the proof of proposition 2.16 .

Remark 2.19. Observe that the function defined by formula 2.8 is not smooth if $g$ is not flat along the subspace $S$. If $g$ is only flat at the origin then we can find examples which show that $f$ does not admit a smooth continuation.

For instance consider $n=4$, the function $g=e^{-\left(\frac{1}{x_{3}}\right)^{2}}$ is flat at the origin but it is not flat along the subspace $S=\left\{\left(0,0, x_{3}, x_{4}\right)\right\}$. Observe that the integral does not extend to a smooth function at points of the form $\left(0, x_{2}^{0}, x_{3}^{0}, x_{4}^{0}\right)$ with $x_{2}^{0} \neq 0$ and $x_{3}^{0} \neq 0$. This integral has been used by some authors without the condition of flatness along the subspace and just the condition of flatness at the origin (see proposition 2.13 in [70]). Thus, the functions defined by those integrals in [70] do not always admit a smooth continuation unless the function $g$ is flat along $S$.

\section{Step 3 Infinitesimal rigidity in the proof of Theorem 2.7}

One may think that a "key point" to obtain rigidity for integrable systems is the existence of compact action (of $\mathbb{T}^{n}$ ).

But, we also have normal forms and rigidity for some non-degenerate singular integrable systems. Indeed we also have infinitesimal rigidity for a certain type of singular integrable systems called non-degenerate. 
In the spirit of Thom and Mather we can study (infinitesimal) stability of an integrable system $F=\left(f_{1}, \ldots, f_{n}\right)$ with additional constraints $\left\{f_{i}, f_{j}\right\}=0$.

For non-degenerate singular integrable systems, we have proved in [57] that a singular Poincaré lemma holds. This results comes from a refinement of the analytical tools explained before and taking into account crossed derivatives.

We denote by $X_{i}$ the Hamiltonian vector fields of with respect to the Darboux symplectic form $\sum d x_{i} \wedge d y_{i}$ of a Williamson basis. We then have,

Theorem 2.20 (Miranda-Vu Ngoc, [57]). Let $g_{1}, \ldots g_{r}$, be a set of germs of smooth functions on $\left(\mathbb{R}^{2 n}, 0\right)$ with $r \leq n$ fulfilling the following commutation relations

$$
X_{i}\left(g_{j}\right)=X_{j}\left(g_{i}\right), \quad \forall i, j \in\{1, \ldots, r\}
$$

where the $X_{i}$ 's are the vector fields defined above. Then there exists a germ of smooth function $G$ and $r$ germs of smooth functions $h_{i}$ such that,

(1) $X_{j}\left(h_{i}\right)=0, \forall i, j \in\{1, \ldots, r\}$.

(2) $g_{i}=h_{i}+X_{i}(G) \forall i \in\{1, \ldots, r\}$.

We want to stress out here that in [20] a similar statement was contained without proof. The problem with the statement in [20] is that the condition $X_{j}\left(f_{i}\right)=0$ was not stated as such but stating that the functions $h_{i}$ were functions of a Williamson basis. There is a problem with this. Take for instance the function,

$$
\psi(x, y)= \begin{cases}e^{-\frac{1}{(x y)^{2}}} & x \geq 0 \\ 2 e^{-\frac{1}{(x y)^{2}}} & x \leq 0\end{cases}
$$

It satisfies $X_{j}\left(h_{i}\right)=0$ for hyperbolic $X_{j}$ but it is not a function of the Williamson basis. This distinction is very relevant in applications to Geometric Quantization with singularities (in [30] an application is given to geometric quantization and the distinction on flat functions not being functions of $x y$ can be read off the formula that counts contributions of hyperbolic singularities to sheaf cohomology).

We can restate this result in a different language.

Theorem 2.21 (Miranda-Vu Ngoc, [57]). Integrable systems are infinitesimally stable in a neighbourhood of a non-degenerate fixed point.

But what do we mean by infinitesimally stable?

We can view an integrable system as a $\mathbb{R}^{n}$ action on $\mathcal{C}^{\infty}(M)$ and in the spirit of Chevalley-Eilenberg [13] we can associate a complex taking into account this action. We also introduced a similar complex in the non-abelian case in [56] to prove infinitesimal rigidity of Hamiltonian actions of compact semisimple type in Poisson manifolds.

Coming back to the integrable case: Let $L \simeq \mathbb{R}^{n}$ be the typical commutative Lie algebra of dimension $n$. $L$ acts on $\mathcal{C}$ by the adjoint representation:

$$
L \times \mathcal{C}^{\infty}(M) \ni(\ell, g) \mapsto\{\mathbf{f}(\ell), g\} \in \mathcal{C}^{\infty}(M) .
$$

We can define a deformation complex using this action of $\mathbb{R}^{n}$ on $\mathcal{C}^{\infty}(M)$. We use the recipe provided by Chevalley-Eilenberg [13] to define this complex. 
The set of cocycles is defined as follows for $q \in \mathbb{N}, C^{q}\left(L, \mathcal{C}^{\infty}(M)\right)=\operatorname{Hom}\left(L^{\wedge q}, \mathcal{C}^{\infty}(M)\right)$ is the space of alternating $q$-linear maps from $L$ to $\mathcal{C}^{\infty}(M)$ and we assume that $C^{0}\left(L, \mathcal{C}^{\infty}(M)\right)=\mathcal{C}^{\infty}(M)$.

The associated differential is denoted by $d_{\mathbf{f}}$. For a 0-cochain $g \in \mathcal{C}^{\infty}(M)$, the 1-cochain $d_{\mathbf{f}}(g)$ is $d_{f}(g)(l)=\{\mathbf{f}(l), g\}, l \in L$ and more generally for a $k$-cochain $\phi$ the $k+1$ cochain $d_{\mathbf{f}}(\phi)$ is

$$
d_{\mathbf{f}}(\phi)\left(l_{1}, \ldots, l_{k+1}\right)=\frac{1}{k+1} \sum_{i=1}^{k+1}(-1)^{i+1}\left\{\mathbf{f}\left(l_{i}\right), \phi\left(\check{l}_{i}\right)\right\}, \lambda_{i} \in L,
$$

where $\check{l_{i}}=\left(l_{1}, \ldots, \check{l_{i}}, \ldots, l_{k+1}\right)$.

Now since $L$ acts trivially on the set of basic functions with respect to the foliation, we can quotient by these basic functions to define the deformation complex (see [57] for more details).

The cocyles $\alpha$ of this deformation complex are defined via $n$ functions $g_{1}=$ $\alpha\left(e_{1}\right), \ldots, g_{n}=\alpha\left(e_{n}\right)$ (mod. basic functions) such that

$$
\forall i, j \quad\left\{g_{i}, f_{j}\right\}=\left\{g_{j}, f_{i}\right\} .
$$

A cocycle defines an infinitesimal deformation of the system since mod $\varepsilon^{2}$ we have

$$
\left\{f_{i}+\varepsilon g_{i}, f_{j}+\varepsilon g_{j}\right\} \equiv 0 .
$$

The our singular Poincaré lemma above can be restated saying that every cocycle is a coboundary and this is what we mean by infinitesimal stability.

Remark 2.22. This singular Poincaré lemma admits a parametrized version. This parametrized version of the singular Poincaré lemma can be used to prove infinitesimal rigidity not only of integrable systems in a neighbourhood of a fixed singular non-degenerate point but also in the neighbourhood of a singular non-degenerate orbit.

2.3. Action-angle coordinates, group actions and rigidity. As a general principle normal forms for geometrical structures give structural stability. Sometimes because of the type of singularities, this is not enough though (as we will see in the Poisson section).

When we have additional symmetries, it is still possible to prove rigidity using the averaging techniques. In this case, we would obtain the equivariant version.

As a first example of this, the equivariant version of Darboux theorem was stated by Weinstein in [74] and was proved by Chaperon in [12] for smooth compact group actions.

In the case the normal forms are given in a neighbhourhood of a fixed point for the action, we may linearize this action in such a way that the normal forms prevail. If the group is non-compact there is a hope to do it for analytic actions of semisimple groups/algebras in the Hamiltonian setting [55]. In the smooth case this is possible only for actions of semisimple actions of compact type [25].

We can also prove the following rigidity theorem for symplectic group actions on a compact symplectic manifold (see [51] and [56]), 
Theorem 2.23. Let $\rho_{0}$ and $\rho_{1}$ be two $C^{2}$-close symplectic actions of a compact Lie group $G$ on a compact symplectic manifold $(M, \omega)$. Then they can be made equivalent by conjugation via a symplectomorphism.

In particular:

- Liouville-Mineur-Arnold theorem and its singular counterpart entails semilocal rigidity for integrable systems in a neighbourhood of a regular compact orbit.

- (equivariant) Darboux theorem gives (equivariant) local rigidity for symplectic forms (for compact group actions).

- We can combine the former rigidity result for symplectic structures together with the stability of the singularities (or regular points) to get an equivariant rigidity result for integrable systems and group actions in symplectic manifolds.

\section{The Contact Case}

Consider a contact manifold $M^{2 n+1}$ together with a contact form. In this section we assume that the Reeb vector field associated to $\alpha$ coincides with the infinitesimal generator of an $S^{1}$ action. We assume further than there exists $n$-first integrals of the Reeb vector field which commute with respect to the Jacobi bracket. Then there are two foliations naturally attached to the situation. On the one hand, we can consider the foliation associated to the distribution generated by the contact vector fields. We call this foliation $\mathcal{F}^{\prime}$. On the other hand we can consider a foliation $\mathcal{F}$ given by the horizontal parts of the contact vector fields. The functions determining the contact vector fields may have singularities. We will always assume that those singularities are of non-degenerate type. Observe that $\mathcal{F}^{\prime}$ is nothing but the enlarged foliation determined by the foliation $\mathcal{F}$ and the Reeb vector field.

Let $\alpha^{\prime}$ be another contact form in a neighbourhood of a compact orbit $\mathcal{O}$ of $\mathcal{F}^{\prime}$ for which $\mathcal{F}$ is Legendrian and such that the Reeb vector field with respect to $\alpha^{\prime}$ coincides with the Reeb vector field associated to $\alpha$. In this section we prove that then there exists a diffeomorphism from a neighbourhood of $\mathcal{O}$ to a model manifold with a fixed rank and Williamson type taking the foliation $\mathcal{F}^{\prime}$ to a linear foliation in the model manifold with a finite group attached to it and taking the initial contact form to the Darboux contact form. As it was done in the last section for Lagrangian foliations determined by a completely integrable system, we can also prove the $G$ equivariant version of this fact for Legendrian foliations. That is, we prove that in the case there exists a compact Lie group preserving the first integrals of the Legendrian foliation and preserving the contact form then the contactomorphism can be chosen to be $G$-equivariant.

The problem of determining normal forms for foliations related to Legendrian foliations has its own story. P. Libermann in [40] established a local equivalence theorem for $\alpha$-regular foliations. Loosely speaking, those foliations are regular foliations containing the Reeb vector field and a Legendrian foliation. The problem of classifying contact forms is different from the problem of classification of contact structures. As a example of this, if $M$ is a compact manifold then any two contact structures are equivalent by deformation as Gray's theorem asserts ([24]). Whereas one can find examples of two contact forms which are not equivalent (see 
for example [23]). The problem of classifying contact structures which are invariant under a Lie group was considered by Lutz in [43]. In particular he proves that two contact structures in a compact manifold $M^{2 n+1}$ which are invariant by a locally free action of $\mathbb{R}^{n+1}$ are equivalent in the sense that there exists an equivariant contactomorphism taking one to the other.

The foliations studied by Libermann and Lutz are regular. The singular counterpart to the result of Lutz was proved by Banyaga and Molino in [4] but for contact forms.

Namely, Banyaga and Molino study the problem of finding normal forms under the additional assumption of transversal ellipticity. The assumption of transversal ellipticity allows to relate the foliation $\mathcal{F}^{\prime}$ of generic dimension $(n+1)$ with the foliation given by the orbits of a torus action.

In this section we extend these results for foliations which are related in the same sense to $(n+1)$-foliations but which are not necessarily identified with the orbits of a torus action. All our study of the problem is done in a neighbourhood of a compact orbit. Global results for contact manifolds admiting torus action have been obtained by Banyaga and Molino in [4] and recently by Lerman in [38]. Linearization results for contact vector fields in $\mathbb{R}^{2 n}$ with an hyperbolic zero were considered by Guillemin and Schaeffer in [26].

The results contained in this section were announced (without proofs) in [50]. We include here an improved version of the proof included in the author's thesis.

Our strategy for the proofs combines the idea of finding a "symplectic slice" which indeed represents the symplectic reduction with respect to the $S^{1}$-action given by the Reeb vector field in the symplectization of the contact manifold. Sometimes, it will be more appropriate to work in the symplectization and descend to the contact manifolds. Others, it will be useful to work on the slice and try to extend results in a neighbourhood of the orbit.

Let us introduce some basic definitions in contact geometry that will be needed later,

Definition 3.1. Let $M^{2 n+1}$ be a $2 n+1$-dimensional manifold. A 1-form on a manifold $M^{2 n+1}$ is a contact form if the set $E=\left\{(p, u) \in T(M), \quad \alpha_{p}(u)=0\right\}$ is a smooth subbundle of $T(M)$ and $d \alpha_{\mid E}$ is a symplectic structure on the vector bundle $E \longrightarrow M$.

When we talk about a contact pair we consider a pair $(M, \alpha)$ where $\alpha$ is a contact form on $M$.

Remark 3.2. The classical definition of contact manifold is the following. It is a pair $(M, \alpha)$ where $\alpha$ satisfies the condition $\alpha \wedge(d \alpha)_{p}^{n} \neq 0, \forall p \in M$. In turn, this condition implies the nonintegrability of the subbundle $E=\left\{(p, u) \in T(M), \quad \alpha_{p}(u)=0\right\}$. That is it is not possible to find a symplectic submanifold $S$ such that $T(S)=E$.

Suppose that $\alpha$ is a contact form on a manifold $M$. Then if $f$ is a positive function the 1-form $f \alpha$ is also a contact form.

This motivates the definition of contact structure, 
Definition 3.3. A contact structure on a manifold $M$ is a subbundle $E$ of the tangent bundle of the form $E=\left\{(p, u) \in T(M), \quad \alpha_{p}(u)=0\right\}$ for some contact form $\alpha$.

In contrast to symplectic manifolds $(M, \omega)$ where the condition $i_{X}(\omega)=0$ implies $X=0$, in a contact manifold we can find non-trivial solutions $X$ to the equation $i_{X}(\omega)=0$. A privileged solution of this equation has the particular name of Reeb vector field. It is a concept attached to the contact form rather than the contact structure.

Definition 3.4. Given a contact pair $(M, \alpha)$, the Reeb vector field $Z$ is the unique vector field satisfying the following two conditions,

- $i_{Z} d \alpha=0$.

- $\alpha(Z)=1$.

The Reeb vector field is a particular case of what we call contact vector field.

Definition 3.5. Let $f$ be a smooth function on the contact pair $(M, \alpha)$ the contact vector field associated to $f$ is the unique vector field $X_{f}$ fulfilling the following two conditions

- $i_{X_{f}} d \alpha_{\mid E}=-d f_{\mid E}$

- $\alpha\left(X_{f}\right)=f$.

Observe that the contact vector field associated to the function 1 is precisely the Reeb vector field. As it is proved in [40], we can express any vector field $X$ in $T(M)$ as a sum of two vector fields $X_{1}$ and $X_{2}$ where the vector field $X_{1}$ belongs to the subbundle $E$ and its called the horizontal part of $X$ and the vector field $X_{2}$ is the component in the direction of the Reeb vector field. The standard notation for the horizontal vector field associated to $X$ is $\widehat{X}$.

We can now define the notion of Jacobi bracket of two functions, which is the contact counterpart to the Poisson bracket of two functions.

Definition 3.6. Let $f, g$ be two smooth functions on a contact pair $(M, \alpha)$, we define the Jacobi bracket as,

$$
[f, g]=\alpha\left(\left[X_{f}, X_{g}\right]\right)
$$

The following relations are proved in [40],

$$
\begin{gathered}
X_{[f, g]}=\left[X_{f}, X_{g}\right] \\
{[f, g]=d \alpha\left(X_{f}, X_{g}\right)+f(Z(g))-g(Z(f))}
\end{gathered}
$$

Definition 3.7. A submanifold $N \subset M^{2 n+1}$ is Legendrian if $\operatorname{dim} N=n$ and $\alpha(X)=0$ for any $X \in T(N)$.

3.1. Contact manifolds and integrability. In this section we define the foliations that we will work with throughout this section and we will also define the linear model. Let $\left(M^{2 n+1}, \alpha\right)$ be a contact pair and let $Z$ be its Reeb vector field. We make the following assumptions, 
- We assume $Z$ coincides with the infinitesimal generator of an $S^{1}$ action. Let $S$ be one of its orbits.

- We assume that there are $n$ first integrals $f_{1}, \ldots, f_{n}$ of $Z$ (that is $Z\left(f_{i}\right)=0$ ) which fulfil the following additional hypotheses:

(1) The first integrals are independent in an open dense set. That is, $d f_{1} \wedge \cdots \wedge d f_{n} \neq 0$ in an open dense set.

(2) The $n$-first integrals are in involution with respect to the Jacobi bracket associated to $\alpha$. That is to say,

$$
\left[f_{i}, f_{j}\right]=0 \quad, \forall i, j .
$$

(3) The minimum rank of the differential $\left(d f_{1}, \ldots, d f_{n}\right)$ is $k$. Let $p$ be a point in $M^{2 n+1}$ such that the rank is exactly $k$. Let $\mathcal{O}$ be the orbit of the contact vector fields through $p$. We will assume the following,

(a) $\mathcal{O}$ is diffeomorphic to a torus of dimension $k+1$.

(b) The first integrals $f_{1}, \ldots, f_{k}$ are non-singular along $\mathcal{O}$ and the first integrals $f_{k+1}, \ldots, f_{n}$ have a non-degenerate singularity in the Morse-Bott sense along $\mathcal{O}$.

Since $\left[f_{i}, f_{j}\right]=0$ then due to formula $3.1,\left[X_{f_{i}}, X_{f_{j}}\right]=0$ and this implies that the distribution $<Z, X_{f_{1}}, \ldots, X_{f_{n}}>$ is involutive because the functions $f_{i}$ are first integrals of the Reeb vector field. Thus, we can talk about the foliation generated by the contact vector fields of the functions $1, f_{1}, \ldots, f_{n}$. This foliation will be denoted by $\mathcal{F}^{\prime}$.

On the other hand, consider the horizontal parts of the contact vector fields. They have the form $\widehat{X}_{f}=X_{f}-f Z$. Thus the distribution $<\widehat{X}_{f_{1}}, \ldots \widehat{X}_{f_{n}}>$ defines an involutive distribution. The foliation defined by this distribution will be denoted by $\mathcal{F}$. Observe that since $\alpha\left(X_{f}\right)=f$ and $\alpha(Z)=1$ then the regular leaves of this foliation are Legendrian submanifolds with respect to $\alpha$.

That is why this foliation will be called the singular Legendrian foliation.

In fact we will work with germ-like foliations. That is, we will assume that the foliation is defined in a neighbourhood of $\mathcal{O}$. Now let $p \in M$ be a singular point. We will say that the point has rank $r$ if the dimension of the orbit through $p$ is $r$.

Once the two foliations $\mathcal{F}$ and $\mathcal{F}^{\prime}$ are defined we are ready to pose the following problem.

\section{Problem}

Study the contact forms $\alpha^{\prime}$ defined in a neighbourhood of $\mathcal{O}$ for which $\mathcal{F}$ is Legendrian and such that the Reeb vector field with respect to $\alpha^{\prime}$ coincides with the Reeb vector field with respect to $\alpha$.

As far as this problem is concerned we will prove the following.

There exists a diffeomorphism $\phi$ defined in a neighbourhood of $\mathcal{O}$ such that $\phi^{*}\left(\alpha^{\prime}\right)=\alpha$ and $\phi$ preserves the foliations $\mathcal{F}$ and $\mathcal{F}^{\prime}$.

In order to deal with this problem we will need to introduce coordinates in such a way that the foliations $\mathcal{F}$ and $\mathcal{F}^{\prime}$ are really simple. This judicious choice of coordinates leads us to the linear model. 
3.1.1. Differentiable linearization. In this subsection we want to prove that under the above assumptions there exist coordinates in a neighbourhood of an orbit of fixed rank and Williamson type $\mathcal{O}$ such that the foliation can be linearized.

We prove the following,

Proposition 3.8. There exist coordinates $\left(\theta_{0}, \ldots, \theta_{k}, p_{1}, \ldots, p_{k}, x_{1}, y_{1}, \ldots, x_{n-k}, y_{n-k}\right)$ in a finite covering of a tubular neighbourhood of $\mathcal{O}$ such that

- The Reeb vector field is $Z=\frac{\partial}{\partial \theta_{0}}$.

- There exists a triple of natural numbers $\left(k_{e}, k_{h}, k_{f}\right)$ with $k_{e}+k_{h}+2 k_{f}=n-k$ and such that the first integrals $f_{i}$ are of the following type, $f_{i}=p_{i}, \quad 1 \leq$ $i \leq k$ and

$$
\begin{aligned}
& f_{i+k}=x_{i}^{2}+y_{i}^{2} \text { for } 1 \leq i \leq k_{e} \\
& f_{i+k}=x_{i} y_{i} \text { for } k_{e}+1 \leq i \leq k_{e}+k_{h}, \\
& f_{i+k}=x_{i} y_{i+1}-x_{i+1} y_{i} \text { and } \\
& f_{i+k+1}=x_{i} y_{i}+x_{i+1} y_{i+1} \text { for } i=k_{e}+k_{h}+2 j-1,1 \leq j \leq k_{f}
\end{aligned}
$$

- The foliation $\mathcal{F}$ is given by the orbits of the distribution $\mathcal{D}=<Y_{1}, \ldots Y_{n}>$ where $Y_{i}=X_{i}-f_{i} Z$ being $X_{i}$ the contact vector field of $f_{i}$ with respect to the contact form $\alpha=d \theta_{0}+\sum_{i=1}^{n-k} \frac{1}{2}\left(x_{i} d y_{i}-y_{i} d x_{i}\right)+\sum_{i=1}^{k} p_{i} d \theta_{i}$.

Proof. First of all, since $Z$ is the infinitesimal generator of an $S^{1}$-action, according to the Slice Theorem [61] a neighbourhood of $\mathcal{O}$ in $M^{2 n+1}$ is diffeomorphic to the bundle $S^{1} \times_{S_{x}^{1}} W$ where $S_{x}^{1}$ denotes the isotropy group at a point in the orbit. Thus we can choose coordinates in a finite covering of a neighbourhood of $\mathcal{O}$ such that the Reeb vector field has the form $Z=\frac{\partial}{\partial \theta_{0}}$. Now the 1 -form $\alpha$ can be written as

$$
\alpha=d \theta_{0}+\bar{\alpha} .
$$

Observe that since $Z$ is the Reeb vector field in particular we obtain

$$
i_{Z} d \bar{\alpha}=0
$$

Using Cartan's formula $L_{Z}(\bar{\alpha})=d i_{Z}(\bar{\alpha})+i_{Z} d \bar{\alpha}$ we deduce that $\bar{\alpha}$ does not depend on $\theta_{0}$.

We now consider the symplectization of neighbourhood of $\mathcal{O}$ which is of type $U(\mathcal{O}) \times(-\varepsilon, \varepsilon)$. The symplectization is endowed with symplectic form $d t \wedge d \theta_{0}+d \bar{\alpha}$.

We denote by $X_{f_{i}}^{s}$ the $n$ Hamiltonian vector fields of the functions $f_{i}$ with respect to the symplectic structure $d t \wedge d \theta+d \bar{\alpha}$ on the symplectization. We denote by $X_{f_{i}}^{c}$ the $n$ contact vector fields of the functions $f_{i}$ with respect to the contact structure $\alpha$. A priori we could write in general

$$
X_{f_{i}}^{c}=X_{f_{i}}^{s}+g_{i} Z
$$

for certain smooth functions $g_{i}$.

We have $\left\{f_{i}, f_{j}\right\}=\left[f_{i}, f_{j}\right]=0$. So we can now consider and consider the integrable system defined by $\left(t, f_{1} \ldots, f_{n}\right)$ in this $2 n+2$ symplectic manifold. As a particular consequence of theorem 2.10, we can assume that this foliation is given by the linear model in this symplectic manifold. Observe that the Reeb vector field is now a Hamiltonian vector field associated to the variable $t$ in this symplectization and it commutes with the other vector fields. Therefore this linearization descends to the initial contact manifold $U(\mathcal{O})$. 
In other words, we can assume that,

$$
\begin{aligned}
& f_{i}=p_{i}, \text { for } 1 \leq i \leq k, f_{i+k}=x_{i}^{2}+y_{i}^{2} \text { for } 1 \leq i \leq k_{e} \\
& f_{i+k}=x_{i} y_{i} \text { for } k_{e}+1 \leq i \leq k_{e}+k_{h} \\
& f_{i+k}=x_{i} y_{i+1}-x_{i+1} y_{i} \text { and } \\
& f_{i+k+1}=x_{i} y_{i}+x_{i+1} y_{i+1} \text { for } i=k_{e}+k_{h}+2 j-1,1 \leq j \leq k_{f}
\end{aligned}
$$

By taking into account how the symplectization is and how the linear model in the symplectic case is. This yields to the notion of model manifold is the manifold $M_{0}^{2 n+1}=\mathbb{T}^{k+1} \times U^{k} \times V^{2(n-k)}$, where $U^{k}$ and $V^{2(n-k)}$ are $k$-dimensional and $2(n-k)$ dimensional disks respectively. Because in the linear model the vector fields are the Hamiltonian vector fields with respect to the functions $f_{i}$ with respect to the symplectic form $\left.d t \wedge d \theta_{0}+\sum_{i=1}^{k} d p_{i} \wedge d \theta_{i}+\sum_{i=1}^{n-k} d x_{i} \wedge d y_{i}\right)$ and those vector fields coincide with the Hamiltonian vector fields with respect to the contact form $\alpha_{0}=d \theta_{0}+\sum_{i=1}^{k} p_{i} d \theta_{i}+\sum_{i=1}^{(n-k)} \frac{1}{2}\left(x_{i} d y_{i}-y_{i} d x_{i}\right)$, we can assume that the conctact form in the model manifold is this one.

The pair $\left(M_{0}^{2 n+1}, \alpha_{0}\right)$ is called the contact model manifold. The Reeb vector field in the contact model manifold is the vector field $\frac{\partial}{\partial \theta_{0}}$.

Now consider functions of the following type, $f_{i}=p_{i}, \quad 1 \leq i \leq k$ and

$$
\begin{aligned}
& f_{i+k}=x_{i}^{2}+y_{i}^{2} \text { for } 1 \leq i \leq k_{e} \\
& f_{i+k}=x_{i} y_{i} \text { for } k_{e}+1 \leq i \leq k_{e}+k_{h} \\
& f_{i+k}=x_{i} y_{i+1}-x_{i+1} y_{i} \text { and } \\
& f_{i+k+1}=x_{i} y_{i}+x_{i+1} y_{i+1} \text { for } i=k_{e}+k_{h}+2 j-1,1 \leq j \leq k_{f}
\end{aligned}
$$

The linear foliation is the foliation given by the orbits of the distribution $\mathcal{D}=<$ $Y_{1}, \ldots Y_{n}>$ where $Y_{i}=X_{i}-f_{i} Z$ being $X_{i}$ the contact vector field of $f_{i}$ in the contact model manifold.

In all, we have proved that there exists a finite covering of a neighbourhood $U(\mathcal{O})$ of the compact orbit considered such that the lifted foliation in the covering is differentially equivalent to the linear foliation in the contact model manifold.

The linear model for the foliation $\mathcal{F}^{\prime}$ is the foliation expressed in the coordinates provided by the theorem together with a finite group attached to the finite covering.

The different smooth submodels corresponding to the model manifold are labeled by a finite group which acts in a contact fashion and preserves the foliation in the model manifold. This is the only differentiable invariant. Therefore, our problem of contact equivalence will be studied in this model manifold and the equivalence will be established via the equivariant version equivalence which will be considered later in this section.

3.1.2. Contact linearization. For the proof of the contact linearization we need to recall the following relative Poincaré lemma. A proof can be found in [74]

Theorem 3.9 (Relative Poincaré lemma). Let $L \subset M$ a closed submanifold and let $\omega$ be a closed $p$-form whose pull-back to $L$ is zero. Then there exists a $p-1$ form $\alpha$ in a neighbourhood of $L$ such $d \alpha=\omega$ and $\alpha$ vanishes on $N$. If $\omega$ vanishes on $L$ then $\phi$ can be chosen so that its first derivatives vanish on $L$. 
We now prove the following theorem,

Theorem 3.10. Let $\alpha$ be a contact form on the model manifold $M_{0}^{2 n+1}$ for which $\mathcal{F}$ is a Legendrian foliation and such that the Reeb vector field is $\frac{\partial}{\partial \theta_{0}}$. Then there exists a diffeomorphism $\phi$ defined in a neighbourhood of the singular orbit of fixed rank and Williamson type $\mathcal{O}=\left(\theta_{0}, \ldots, \theta_{k}, 0, \ldots, 0\right)$ preserving $\mathcal{F}^{\prime}$ and taking $\alpha$ to $\alpha_{0}$.

Proof. We are going to solve the problem by adjusting the contact form to a point where we can apply our symplectic linearization result.

Let us start by considering the contact 1 -form $\alpha$,

$$
\alpha=A d \theta_{0}+\sum B_{i} d p_{i}+\sum C_{i} d \theta_{i}+\sum D_{i} d x_{i}+\sum E_{i} d y_{i}
$$

Observe that the fact that the Reeb vector field is $\frac{\partial}{\partial \theta_{0}}$ imposes the following two conditions on $\alpha$,

- $\alpha\left(\frac{\partial}{\partial \theta_{0}}\right)=1$, that is to say $A=1$.

So far we can write $\alpha=d \theta_{0}+\alpha^{\prime}$, being $\alpha^{\prime}=\sum B_{i} d p_{i}+\sum C_{i} d \theta_{i}+$ $\sum D_{i} d x_{i}+\sum E_{i} d y_{i}$

- $i_{\frac{\partial}{\partial \theta_{0}}} d \alpha=0$,

Since $d \alpha=d \alpha^{\prime}$ the condition becomes, $i_{\frac{\partial}{\partial \theta_{0}}} d \alpha^{\prime}=0$.

Now Cartan's formula yields,

$$
0=i_{\frac{\partial}{\partial \theta_{0}}} d \alpha^{\prime}=L_{\frac{\partial}{\partial \theta_{0}}} \alpha^{\prime}-d i_{\frac{\partial}{\partial \theta_{0}}} \alpha^{\prime}
$$

Since the last term vanishes this chain of equalities give the condition $L_{\frac{\partial}{\partial \theta_{0}}} \alpha^{\prime}=0$.

Therefore, the coefficient functions do not depend on $\theta_{0}$. Let us see that the submanifold $\theta_{0}=0$ equipped with the form $d \alpha^{\prime}$ is a symplectic submanifold of the model contact manifold. We denote this submanifold by $N$.

Since $\alpha$ is a contact form $d \alpha$ has to be symplectic in the vector bundle $E$ defined by $E=\left\{(p, u) \in T(M), \quad \alpha_{p}(u)=0\right\}$ and $d \alpha=d \alpha^{\prime}$ then $d \alpha^{\prime}$ defines a symplectic structure on $N{ }^{4}$

Observe that the vector fields $X_{i}=X_{f_{i}}$ are tangent to the submanifold $N$. Next step, we check that the vector fields $X_{i}$ are define a Lagrangian foliation on $N$, observe that $\alpha\left(X_{i}\right)=f_{i}$.

Now since, $d \alpha^{\prime}\left(X_{i}, X_{j}\right)=X_{i} \alpha\left(X_{j}\right)-X_{j} \alpha\left(X_{i}\right)-\alpha\left(\left[X_{i}, X_{j}\right]\right)$

According to the computation above $X_{i} \alpha\left(X_{j}\right)=X_{i}\left(f_{j}\right)$ but $f_{i}$ are first integrals for the foliation and therefore this term vanishes. Symmetrically, the second term vanishes. And since the Lie bracket of the vector fields are zero we obtain,

$$
d \alpha^{\prime}\left(X_{i}, X_{j}\right)=0
$$

Therefore, the foliation $\mathcal{F}$ is Lagrangian for $d \alpha^{\prime}$ and we may apply the symplectic linearization result in a neighbourhood of $L=\mathbb{T}^{k}$ (theorem 2.10) to find a local diffeomorphism $\varphi: U(L) \longrightarrow \varphi(U(L))$ in a neighbourhood of the leaf $L$, preserving

\footnotetext{
"Observe that $N$ is indeed a "symplectic slice" which can be identified with the symplectic reduction by the $\mathbb{S}^{1}$-action given by the Reeb vector field on the symplectization.
} 
the foliation $\mathcal{F}$ and satisfying $\varphi^{*}\left(\omega_{0}\right)=d \alpha^{\prime}$, where $\omega_{0}=\sum_{i} d p_{i} \wedge d \theta_{i}+\sum d x_{i} \wedge d y_{i}$. After shrinking the initial neighbourhood if necessary, the neighbourhood of $\mathbb{T}^{k+1}$ in the initial manifold $M$ can be decomposed as a product, $\mathbb{S}^{1} \times U(L)$. The $\mathbb{S}^{1}$ corresponds to an orbit of the Reeb vector field. We denote by $z$ a point in $U(L)$. Now we define a diffeomorphism in the following way,

$$
\begin{aligned}
& \phi: \quad \mathbb{S}^{1} \times U(L) \longrightarrow \phi\left(\mathbb{S}^{1} \times U(L)\right) \\
& \left(\theta_{0}, z\right) \quad \longrightarrow \quad\left(\theta_{0}, \varphi(z)\right)
\end{aligned}
$$

Since $\varphi$ preserves $\mathcal{F}$ it is clear that this diffeomorphism is foliation-preserving.

Now consider $\phi\left(\mathbb{S}^{1} \times U(L)\right)$ endowed with the Darboux contact form. That is with the contact form $\alpha_{0}=d \theta_{0}+\sum_{i=1}^{k} p_{i} d \theta_{i}+\sum_{i=1}^{(n-k)} \frac{1}{2}\left(x_{i} d y_{i}-y_{i} d x_{i}\right)$. It remains to check that the diffeomorphism above is indeed a contactomorphism.

First observe that since

$$
\varphi^{*}\left(\omega_{0}\right)=d \alpha^{\prime}
$$

and $\omega_{0}=d(\beta)$, being $\beta=\left(\sum_{i=1}^{k} p_{i} d \theta_{i}+\sum_{i=1}^{(n-k)} \frac{1}{2}\left(x_{i} d y_{i}-y_{i} d x_{i}\right)\right)$ we can assert that $\varphi^{*}(\beta)=\alpha^{\prime}+d H+\sum_{i} H_{i}(0) d \theta_{i}$ for a set of smooth functions $H$ and $H_{i}\left(\theta_{i}\right)$.

We can decompose the functions $H_{i}(\theta)=H(0)+\frac{\partial M_{i}}{\partial \theta}$ for certain functions $M_{i}$ depending on $\theta_{i}$ so we may gather these terms into $d \bar{H}$ and therefore we can write,

$$
\varphi^{*}(\beta)=\alpha^{\prime}+d \bar{H}+\sum_{i} H_{i}\left(\theta_{i}\right) d \theta_{i} .
$$

Now because of relative Poincaré lemma 3.9, we can assume that the one form $d \bar{H}+\sum_{i} H_{i}(0) d \theta_{i}$ vanishes on $L$ and thus $H_{i}(0)=0$.

Now consider the path $\alpha_{t}=\alpha_{0}+t d \bar{H}$ being $\alpha_{0}$ the contact form $\alpha_{0}=d \theta_{0}+\alpha^{\prime}$. Consider

$\psi_{1}\left(\theta_{0}, \theta_{1}, \ldots, \theta_{k}, p_{1}, \ldots, p_{k}, x_{1}, \ldots, y_{n-k}\right)=\left(\theta_{0}-\bar{H}, \theta_{1}, \ldots, \theta_{k}, p_{1}, \ldots, p_{k}, x_{1}, \ldots, y_{n-k}\right)$, we obtain $\psi_{1}^{*}\left(\alpha_{1}\right)=\alpha_{0}$.

Therefore $\phi$ is a contactomorphism and clearly it preserves the foliation.

Remark 3.11. The proof we have included here is the one provided in the author's thesis [49]. There is another way to go which is to consider the reduction via the Hamiltonian $\mathbb{S}^{1}$-action that the Reeb vector field determines in its symplectization as we did to prove differentiable linearization. In the next section we use this idea to prove an equivariant statement of the theorem above. Observe that the symplectic manifold $N$ in this proof can be indeed identified with a symplectic reduction of the $\mathbb{S}^{1}$ action in the symplectization.

3.2. Additional symmetries and rigidity. In this subsection we consider a compact Lie group $G$ acting on a contact model manifold in such a way that preserves the $n$ first integrals of the Reeb vector field and preserves the contact form as well. We want to prove that there exists a diffeomorphism in a neighbourhood of $\mathcal{O}$ preserving the $n$ first integrals , preserving the contact form and linearizing the action of the group. This result is a consequence of the equivariant symplectic linearization theorem. 
The notion of linear action of a Lie group on the contact model manifold is analogous to the equivalent notion for the symplectic model manifold.

Let $G$ be a group defining a smooth action $\rho: G \times M_{0}^{2 n+1} \longrightarrow M_{0}^{2 n+1}$ on $M_{0}^{2 n+1}$. We assume that this action preserves the contact form $\alpha_{0}$ of the contact model manifold. That is to say $\rho_{g}^{*}\left(\alpha_{0}\right)=\alpha_{0}$. Assume further that it preserves the $n$-first integrals $\left(f_{1}, \ldots, f_{n}\right)$, where $f_{i}=p_{i}, \quad 1 \leq i \leq k$. For the sake of simplicity we denote by $F$ the collective mapping $F=\left(p_{1}, \ldots, p_{k}, f_{k+1}, \ldots, f_{n}\right)$. We will say that the action of $G$ on $M_{0}^{2 n+1}$ is linear if it satisfies the following property:

$G$ acts on the product $M_{0}^{2 n+1}=D^{k} \times \mathbb{T}^{k+1} \times D^{2(n-k)}$ componentwise; the action of $G$ on $D^{k}$ is trivial, its action on $\mathbb{T}^{k+1}$ is by translations (with respect to the coordinate system $\left.\left(\theta_{0}, \ldots, \theta_{k}\right)\right)$, and its action on $D^{2(n-k)}$ is linear with respect to the coordinate system $\left(x_{1}, y_{1}, \ldots, x_{n-k}, y_{n-k}\right)$.

Under the above notations and assumptions. Now we can state and prove the following theorem,

Theorem 3.12. There exists a diffeomorphism $\phi$ defined in a tubular neighbourhood of $\mathcal{O}$ such that,

- it preserves the contact form $\alpha_{0}$ i.e $\phi^{*}\left(\alpha_{0}\right)=\alpha_{0}$.

- it preserves $F$.

- it linearizes the action of $G$. That is to say $\phi \circ \rho_{g}=\rho_{g}^{(1)} \circ \phi$.

Proof. Recall that $\alpha_{0}=d \theta_{0}+\bar{\alpha}_{0}$ being $\bar{\alpha}_{0}$ the 1-form $\left(\sum_{i=1}^{k} p_{i} d \theta_{i}+\sum_{i=1}^{(n-k)} \frac{1}{2}\left(x_{i} d y_{i}-\right.\right.$ $\left.y_{i} d x_{i}\right)$ ). Consider the symplectic manifold $S=M_{0}^{2 n+1} \times(-\varepsilon, \varepsilon)$ endowed with the symplectic form $\omega_{0}=d t \wedge d \theta_{0}+d \bar{\alpha}_{0}$, where $t$ stands for a coordinate function on $(-\varepsilon, \varepsilon)$. An action of $G$ on $M_{0}^{2 n+1}$ can be extended in a natural way to an action of $G$ on $S$ as follows,

$$
\begin{array}{ccc}
\widehat{\rho}: \quad G \times M_{0}^{2 n+1} \times(-\varepsilon, \varepsilon)+ & \longrightarrow & M_{0}^{2 n+1} \times(-\varepsilon, \varepsilon) \\
(g, z, t) & \longrightarrow & \left(\rho_{g}(z), t\right)
\end{array}
$$

On $S$ we consider the moment mapping $\widehat{F}=(F, t)$. We can apply the equivariant linearization theorem to obtain a symplectomorphism $\widehat{\varphi}$ preserving $\widehat{F}$ and linearizing the action $\widehat{\rho}$. From the definition of the action $\widehat{\rho}$ and the definition of $\widehat{F}$, this symplectomorphism clearly descends to a diffeomorphism $\varphi$ on $M_{0}^{2 n+1}$ which linearizes the action $\rho$ and which satisfies $\varphi^{*}\left(d \alpha_{0}\right)=d \alpha_{0}$.

Therefore we may assume $\varphi^{*}\left(\alpha_{0}\right)=\alpha_{O}+d f+\sum_{i} H_{i}\left(\theta_{i}\right) d \theta_{i}$ for a set of smooth functions $f$ and $H_{i}\left(\theta_{i}\right)$.

We can decompose the functions $H_{i}(\theta)=H(0)+\frac{\partial M_{i}}{\partial \theta}$ for certain functions $M_{i}$ depending on $\theta_{i}$ so we may gather these terms into $d \bar{H}$ and therefore we can write,

$$
\varphi^{*}\left(\alpha_{0}\right)=\alpha_{0}+d \bar{H}+\sum_{i} H_{i}\left(\theta_{i}\right) d \theta_{i} .
$$

Now because of relative Poincaré lemma 3.9, we can assume that the one form $\alpha_{0}+d \bar{H}+\sum_{i} H_{i}(0) d \theta_{i}$ vanishes on $L$ an therefore $H_{i}(0)=0$.

Giving, 


$$
\varphi^{*}\left(\alpha_{0}\right)=\alpha_{0}+d \bar{H}
$$

Finally the diffeomorphism,

$$
\phi\left(\theta_{0}, \ldots, \theta_{k}, p_{1}, \ldots, p_{k}, x_{1}, \ldots, y_{n-k}\right)=\left(\theta_{0}-\bar{H}, \ldots, \theta_{k}, p_{1}, \ldots, p_{k}, x_{1}, \ldots, y_{n-k}\right)
$$

takes the form $\alpha_{0}+d h$ to $\alpha_{0}$ and provides new coordinates for which the action is linear.

In the previous section we have attained the contact linearization in the covering. Now applying the theorem of equivariant linearization to the group of deck transformations we obtain as a corollary the following theorem,

Theorem 3.13. Let $\mathcal{F}$ be a foliation fulfilling the hypotheses specified in section 3.1, let $\mathcal{F}^{\prime}$ be the enlarged foliation with the Reeb vector field $Z$ and let $\alpha$ be a contact form for which $\mathcal{F}$ is Legendrian and such that $Z$ is the Reeb vector field then there exists a diffeomorphism defined in a neighbourhood of fixed rank and Williamson type, $\mathcal{O}$ taking $\mathcal{F}^{\prime}$ to the linear foliation, the orbit $\mathcal{O}$ to the torus $\left\{x_{i}=\right.$ $\left.0, y_{i}=0, p_{i}=0\right\}$ and taking the contact form to the Darboux contact form $\alpha_{0}$.

3.2.1. Another approach to the equivariant case: The rigidity problem. In the same sense, that the rigidity problem was approached in the symplectic case, we can also prove that close contact structures are equivalent at the local, semilocal and global case in the

The local case a linearization result for compact contact group actions was already established by Marc Chaperon [12].

In the global case, we can use the path method in the contact setting due to [24] and reproduce the same ideas of the proof of the symplectic case.

This statement is implicit in [56],

Theorem 3.14 (Miranda-Monnier-Zung). Let $\rho_{0}$ and $\rho_{1}$ be two $C^{2}$-close contact actions of a compact Lie group $G$ on a compact contact manifold $(M, \alpha)$. Then they can be made equivalent by conjugation via a contactomorphism.

\section{The Poisson CASE}

In this section we are going to provide some normal form results for integrable systems in the Poisson setting. We start by defining what is a Poisson structure.

When working with Poisson structure we need to work with bivector fields instead of using forms.

Definition 4.1. A Poisson structure on a smooth manifold $M$ is given by a smooth antisymmetric bivector field $\Pi$ satisfying $[\Pi, \Pi]=0$.

This defines a Poisson bracket on $\mathcal{C}^{\infty}(M)$,

$$
\{f, g\}:=\Pi(d f, d g)
$$


Hamiltonian vector fields are defined by the formula $X_{f}:=\Pi(d f, \cdot)$ and the manifold $M$ is endowed with a smooth foliation (in the Sussmann sense) whose leaves are symplectic manifolds. This symplectic foliation integrates the distribution of Hamiltonian vector fields.

There is no Darboux theorem for Poisson manifolds. The best "normal form" that we can get is the following result due to Weinstein [75]

Theorem 4.1 (Weinstein). Let $\left(M^{n}, \Pi\right)$ be a smooth Poisson manifold and let $p$ be a point of $M$ of rank $2 k$, then there is a smooth local coordinate system $\left(x_{1}, y_{1}, \ldots, x_{2 k}, y_{2 k}, z_{1}, \ldots, z_{n-2 k}\right)$ near $p$, in which the Poisson structure $\Pi$ can be written as

$$
\Pi=\sum_{i=1}^{k} \frac{\partial}{\partial x_{i}} \wedge \frac{\partial}{\partial y_{i}}+\sum_{i j} f_{i j}(z) \frac{\partial}{\partial z_{i}} \wedge \frac{\partial}{\partial z_{j}}
$$

where $f_{i j}$ vanish at the origin.

In other words, the Poisson manifold is locally a product of a symplectic manifold with a Poisson manifold with vanishing Poisson structure at the point.

$$
\left(M^{n}, \Pi, p\right) \approx\left(N^{2 k}, \omega, p_{1}\right) \times\left(M_{0}^{n-2 k}, \Pi_{0}, p_{2}\right)
$$

The symplectic foliation on the manifold is locally a product of the induced symplectic foliation on $M_{0}$ with the symplectic leaf through $x$.

Let us start by defining what is an integrable system on a Poisson manifold.

Definition 4.2. Let $(M, \Pi)$ be a Poisson manifold of (maximal) rank $2 r$ and of dimension $n$. An $s$-tuplet of functions $\mathbf{F}=\left(\mathbf{f}_{\mathbf{1}}, \ldots, \mathbf{f}_{\mathbf{s}}\right)$ on $M$ is said to define a Liouville integrable system on $(M, \Pi)$ if

(1) $f_{1}, \ldots, f_{s}$ are independent

(2) $f_{1}, \ldots, f_{s}$ are pairwise in involution

(3) $r+s=n$

Viewed as a map, $\mathbf{F}: \mathbf{M} \rightarrow \mathbf{R}^{\mathbf{s}}$ is called the momentum map of $(M, \Pi, \mathbf{F})$.

There are several problems analogous to the symplectic case that we could consider in the Poisson setting: local normal forms and action-angle coordinates.

We start this section by providing some natural examples of Hamiltonian and integrable systems in Poisson manifolds.

\subsection{Motivating examples.}

4.1.1. Example 1: Newton systems. This example was found together with Alain Albouy. We thank him for explaining Projective Dynamics.

Consider a system of the form:

$$
\ddot{q}=f(q)
$$

These kind of systems are called Newton systems. It is a Hamiltonian system in moment and position coordinates.Appell discovered that such systems can be projectivised. Appell's transformation (central projection) allows to change the 
"screen" of projection (change of affine coordinates). Two such systems and their solutions are equivalent. This is the principle of "Projective Dynamics" (Appell, Killing, Albouy [1]...).

The study of projective dynamics allows to:

- Solve some problems by separation of variables.

- Simplify the solution of those systems by finding an appropriate screen. For instance the Neumann problem on the ellipsoid becomes a Newton System (Knoerrer).

By means of Appell's transformation we transform both the dynamics and the initial symplectic structure. We are including singularities into the picture (coming from projectivisation). It motivates to look at the integrable system from the Poisson point of view.

An example of Newton system is the two fixed-center problem (Euler, 1760). A particle in the plane moves under the gravitational attraction of two fixed points $A$ and $B$ with masses $m_{A}$ and $m_{B}$.

This system reads:

$$
\ddot{q}=-m_{A} \frac{q_{A}}{\left\|q_{A}\right\|^{3}}-m_{B} \frac{q_{B}}{\left\|q_{B}\right\|^{3}}
$$

$\left(q_{A}=q-A, q_{B}=q-B.\right)$ Two first integrals are:

$$
\begin{gathered}
H=\frac{1}{2}\|\dot{q}\|^{2}-\frac{m_{A}}{\left\|q_{A}\right\|}-m_{B} \frac{m_{B}}{\left\|q_{B}\right\|} \\
G=\left\langle q_{A} \wedge \dot{q}, q_{B} \wedge \dot{q}\right\rangle-\frac{m_{A}}{\left\|q_{A}\right\|}\left\langle q_{A}, u\right\rangle-m_{B} \frac{m_{B}}{\left\|q_{B}\right\|}\left\langle q_{B}, u\right\rangle
\end{gathered}
$$

where $u=q_{A}-q_{B}$

They satisfy $\{H, G\}=0$. We can now perform central projection for the twocenter problem:

We start from the cotangent bundle in $T^{*}\left(\mathbb{R}^{2}\right)$. Consider the "position" homogeneous coordinates $\left[q_{0}: q_{1}: q_{2}\right]$. (the initial affine chart is $q_{0}=1$ ).

We now perform central projection to the screen $q_{2}=1$. After this, we change the momenta accordingly and we obtain an integrable system on the new screen.

The new "symplectic"structure (it is not symplectic we added "singularities" in the procedure) reads:

$d v_{1} \wedge d q_{1}+\frac{q_{1}}{q_{2}}\left(d q_{1} \wedge d v_{2}+d q_{2} \wedge d v_{1}\right)+\frac{\left(v_{2} q_{1}-v_{1} q_{2}\right)}{q_{2}^{2}} d q_{1} \wedge d q_{2}+\left(\frac{q_{1}^{2}}{q_{2}^{2}}-1\right) d v_{2} \wedge d q_{2}$

It makes sense to dualize the 2 -form to get the hyperplane $q_{2}=0$ into the picture. By doing so, we can associate a bivector field which is a Poisson structure on a dense set together with an integrable system on it.

4.1.2. Example 2: Integrable systems on b-symplectic manifolds. There is a special class of Poisson structure which share many particularities with the symplectic case. These are called $b$-symplectic or $b$-Poisson structure. 
Definition 4.2. Let $\left(M^{2 n}, \Pi\right)$ be an oriented Poisson manifold such that the map

$$
p \in M \mapsto(\Pi(p))^{n} \in \Lambda^{2 n}(T M)
$$

is transverse to the zero section, then $Z=\left\{p \in M \mid(\Pi(p))^{n}=0\right\}$ is a hypersurface and we say that $\Pi$ is a $b$-Poisson structure on $(M, Z)$ and $(M, Z)$ is a $b$-Poisson manifold. The hypersurface $Z$ is called critical set.

These manifolds have a symplectic foliation which consists on a dense symplectic leaf and the rest of the symplectic leaves are of codimension 2 and lie on the critical subset $Z$ (indeed these codimension 2 leaves define the (codimension 1 ) symplectic foliation of the induced Poisson structure $\Pi_{Z}$.

The study of the geometry of $b$-symplectic manifolds started with the works of Nest and Tsygan on deformation quantization of symplectic manifolds with boundary [60]. We have recently studied the symplectic and Poisson geometry of these manifolds in [28], [29] together with Victor Guillemin and Ana Rita Pires.

The following is an example of integrable system on $b$-Poisson manifold. Consider as $b$-Poisson manifold $\mathbb{R}^{2 n}$ with coordinates $\left(x_{1}, y_{1}, \ldots, x_{n-1}, y_{n-1}, z, t\right) \Pi=$ $\sum_{i=1}^{n-1} \frac{\partial}{\partial x_{i}} \wedge \frac{\partial}{\partial y_{i}}+z \frac{\partial}{\partial z} \wedge \frac{\partial}{\partial t}$.

Observe that the functions $f_{i}=x_{i} \forall i \leq n-1$ and $f_{n}=z$ are pairwise in involution. Thus, these functions define an integrable system $\mathbf{F}=\left(x_{1}, \ldots, x_{n-1}, z\right)$.

As it is seen in [29], the Poisson geometry of the manifold can be reconstructed semilocally from the critical hypersurface $Z$ (a codimension one symplectic foliation admitting a transverse Poisson vector field). We can use a similar strategy to give natural global examples of integrable systems on $b$-Poisson manifold (which includes the compact case).

For instance, consider the following construction: Take an integrable system on a symplectic manifold $(M, \omega, \mathbf{F})$ and consider a symplectomorphism $\phi$ preserving the integrable system. We may consider the symplectic mapping torus associated to this symplectomorphism

$$
N^{2 n+1}=\frac{M \times[0,1]}{(x, 0) \sim(\phi(x), 1)} .
$$

This produces a family of integrable systems on the fibers of the symplectic mapping torus which has naturally the structure of a Poisson structure $\pi$. There is a vector field $X$ transverse to the symplectic manifold $M$ and which preserve the integrable system and this Poisson structure.

Let $f: \mathbb{S}^{1} \rightarrow \mathbb{R}$ a smooth function. The bivector field

$$
\Pi=f(\theta) \frac{\partial}{\partial \theta} \wedge X+\pi
$$

is a $b$-symplectic structure on $\mathbb{S}^{1} \times N$ if the function $f$ vanishes linearly (the manifold has as many critical components as the number of zeroes of the function $f$ ). Also the new system $\mathbf{H}=(f, \mathbf{F})$ is an integrable system on the $b$-symplectic manifold.

As a particular example, take $(M, \omega)=\left(\mathbb{R}^{2 n-2}, \sum_{i=1}^{n-1} d x_{i} \wedge d y_{i}\right)$ and consider the integrable system defined by the moment map $\mathbf{F}=\left(x_{1}, \ldots, x_{n-1}\right)$. Now consider the direct product of $\mathbb{R}^{2 n-2}$ with $T^{*}\left(S^{1}\right)$ with parallelization given by $(\theta, z)$ and 
consider the function $f(\theta)=\sin (\theta)$. The system defined by

$$
\mathbf{F}=\left(z, x_{1}, \ldots, x_{n-1}\right)
$$

on the product

$$
T^{*}\left(S^{1}\right) \times \mathbb{R}^{2 n-2}
$$

endowed with the $b$-Poisson structure,

$$
\Pi=\sin (\theta) \frac{\partial}{\partial \theta} \wedge \frac{\partial}{\partial z}+\sum_{i=1}^{n-1} \frac{\partial}{\partial x_{i}} \wedge \frac{\partial}{\partial y_{i}}
$$

is an integrable system of this type with a trivial mapping torus given by a product also observe that $\frac{\partial}{\partial z}$ leaves the integrable system and is transverse to the symplectic foliation defined by $z=c t$. Observe that for $\sin (\theta)$ vanishes for $\theta=0$ and $\theta=\pi$. That is to say, the critical manifold of the $b$-Poisson manifold in this example has two connected components.

More generally, we could replace symplectic mapping torus construction by a $\left(N^{2 n+1}, \pi\right)$ be a regular corank-1 Poisson manifold and consider $X$ to be a Poisson vector field.

As we will see in a future work, this example is indeed a "canonical"model because we have Moser normal forms for these manifolds.

4.1.3. Example 3: Gelfand-Cetlin system. One of the most basic examples of Poisson manifolds is that of dual of Lie algebras $\mathfrak{g}^{*}$. The symplectic foliation is given by its coadjoint orbits which are endowed with the Kostant-Kirillov-Souriau symplectic form.

The Gelfand-Cetlin system has been classically (Guillemin-Sternberg) considered as an integrable system on a coadjoint orbit $\mathcal{O}$ of $\mathfrak{u}(n)^{*}$. A good reference for this system is [27].

The dual of a Lie algebra constitute a simple example of linear Poisson structure with Poisson brackets defined via the structure constants. The Gelfand-Cetlin system can be seen as a system on the dual of a Lie algebra, as follows. We dualize the increasing sequence of Lie algebra inclusions:

$$
\mathfrak{u}(1) \subset \cdots \subset \mathfrak{u}(n-1) \subset \mathfrak{u}(n)
$$

where $\mathfrak{u}(k)$ is considered as the left-upper diagonal block of $\mathfrak{u}(k+1)$ for $k=1, \ldots, n-$ 1 ), we get a sequence of surjective Poisson maps:

$$
\mathfrak{u}(n)^{*} \longmapsto \mathfrak{u}(n-1)^{*} \longmapsto \cdots \longmapsto \mathfrak{u}(1)^{*}
$$

The family of functions on $\mathfrak{u}(n)^{*}$ obtained by pulling-back generators of the Casimir algebras of all the $\mathfrak{u}(k)^{*}$ for $k=1, \ldots, n$ yields an integrable system on $\mathfrak{u}(n)^{*}$. The complete integrability of this system is a consequence of the method of Thim.

For particular generators, its restriction to an open subset of $\mathcal{O}$ gives the GelfandCetlin system (a commendable reference for this paper is [27]).

This system is defined not only when restricted to the coadjoint orbit with the Kirillov-Kostant-Souriau symplectic structure but on $\mathfrak{u}(n)^{*}$. 
4.1.4. Example 4: Magnetic Flows on Homogeneous Spaces and coadjoint orbits. Let $G$ be a compact Lie group and $H$ a closed subgroup. Let $a \in \mathfrak{h}$ be $H$-adjoint invariant. This implies that $H$ is contained in the isotropy group $G_{a}$. Denote by $\mathcal{O}(a)$ the adjoint orbit. We then have a submersion of homogeneous spaces $\sigma: G / H \longrightarrow G / G_{a} \cong \mathcal{O}(a)$ and $\omega=\sigma^{*}\left(\Omega_{K K S}\right)$ (with $\Omega_{K K S}$ the Kostant-KirillovSouriau symplectic form) is a closed 2 -forms and gives a magnetic field on $G / H$.

We then have [5]:

Theorem 4.3 (Bolsinov, Jovanovich). The magnetic geodesic flows of normal metric $d s_{0}^{2}$ in $G / H$ with respect to the magnetic form $\omega$ is completely integrable in the non-commutative sense.

By using a Theorem of Mischenko-Fomenko which states that non-commutative integrable Hamiltonian systems are also commutatively integrable we obtain an example of integrable system: The magnetic geodesic flow on $G / H$. This system can be viewed in the Poisson manifold $T^{*}(G) / H$.

4.2. A Darboux-Carathéodory theorem in the Poisson context. We start by stating the local normal theorem that we have for Poisson structures contained in $[37]$ :

Theorem 4.4 (Laurent, Miranda, Vanhaecke [37]). Let $m$ be a point of a Poisson manifold $(M, \Pi)$ of dimension $n$. Let $p_{1}, \ldots, p_{r}$ be $r$ functions in involution, defined on a neighborhood of $m$, which vanish at $m$ and whose Hamiltonian vector fields are linearly independent at $m$. There exist, on a neighborhood $U$ of $m$, functions $q_{1}, \ldots, q_{r}, z_{1}, \ldots, z_{n-2 r}$, such that

(1) The $n$ functions $\left(p_{1}, q_{1}, \ldots, p_{r}, q_{r}, z_{1}, \ldots, z_{n-2 r}\right)$ form a system of coordinates on $U$, centered at $m$;

(2) The Poisson structure $\Pi$ is given on $U$ by

$$
\Pi=\sum_{i=1}^{r} \frac{\partial}{\partial q_{i}} \wedge \frac{\partial}{\partial p_{i}}+\sum_{i, j=1}^{n-2 r} g_{i j}(z) \frac{\partial}{\partial z_{i}} \wedge \frac{\partial}{\partial z_{j}},
$$

where each function $g_{i j}(z)$ is a smooth function on $U$ and is independent of $p_{1}, \ldots, p_{r}, q_{1}, \ldots, q_{r}$.

Observe that in this theorem we have adapted Weinstein's splitting theorem to some of the first integrals of the integrable system but not all of them.

4.3. Split systems and rigidity. As we explained in [37], not every integrable system on a Poisson manifold can be split (in a compatible way with Weinstein's theorem).

The following family of counter-examples is contained in [37]:

On $\mathbf{R}^{4}$, with coordinates $f_{1}, f_{2}, g_{1}, g_{2}$, consider the Poisson structure:

$$
\Pi=\frac{\partial}{\partial f_{1}} \wedge \frac{\partial}{\partial g_{1}}+F\left(g_{2}\right) \frac{\partial}{\partial f_{2}} \wedge \frac{\partial}{\partial g_{2}}+G\left(g_{2}\right) \frac{\partial}{\partial g_{1}} \wedge \frac{\partial}{\partial f_{2}},
$$

with vanishing $F$ and $G$ at the origin $g_{2}=0$. 
The pair of functions $\mathbf{F}=\left(f_{1}, f_{2}\right)$ define an integrable system on $\mathbb{R}^{4}$ endowed with this Poisson structure.

As we proved in [37] whenever the expression $\frac{F(z)}{G(z)}$ does not admit a smooth continuation at 0 . the system defined by $F$ is not split. A system is not split whenever we cannot find coordinates in which we have Weinstein's splitting coordinates adapted to the integrable system.

In particular, for the choices if $F(z)=g_{2}^{2}$ and $G_{\varepsilon}(z)=\varepsilon g_{2}$, the system defined by $\mathbf{F}=\left(f_{1}, f_{2}\right)$ is not split.

Indeed the fact, that these systems cannot be split is closely related to the nonrigidity of integrable systems in the Poisson context. That is to say, we can find close integrable systems which are not equivalent. The key point in this proof is the non-stability phenomena present in Poisson geometry.

In order to do this observe consider the family of Poisson structures:

$$
\Pi_{\varepsilon}=\frac{\partial}{\partial f_{1}} \wedge \frac{\partial}{\partial g_{1}}+g_{2}^{2} \frac{\partial}{\partial f_{2}} \wedge \frac{\partial}{\partial g_{2}}+\varepsilon g_{2} \frac{\partial}{\partial g_{1}} \wedge \frac{\partial}{\partial f_{2}},
$$

For $\varepsilon=0$ the system defined by $\mathbf{F}=\left(f_{1}, f_{2}\right)$ is clearly split with respect to the Poisson structure $\Pi_{0}$ (the system splits in Weinstein coordinates) but for $\varepsilon \neq 0$ the system $\mathbf{F}=\left(f_{1}, f_{2}\right)$ is not split with respect to the Poisson structure $\Pi_{\varepsilon}$ as guaranteed from the conditions specified above on $F$ and $G$.

Now consider the local diffeomorphism $\phi_{\varepsilon}\left(f_{1}, f_{2}, g_{1}, g_{2}\right)=\left(f_{1}, f_{2}+\varepsilon g_{2} f_{1}, g_{1}, g_{2}\right)$. This diffeomorphism satisfies $\phi_{\varepsilon, *}\left(\Pi_{0}\right)=\Pi_{\varepsilon}$ but the diffeomorphism does not preserve the integrable system defined by $\left(f_{1}, f_{2}\right)$. Now consider a new integrable system defined via,

$$
\mathbf{F}_{\varepsilon}=\phi_{*}\left(f_{1}, f_{2}\right)=\left(f_{1}, f_{2}+\varepsilon g_{2} f_{1}\right)
$$

This integrable system (which is close to the initial one) is clearly not equivalent to it because it is split with respect to $\Pi_{\varepsilon}$ (since $\mathbf{F}=\left(f_{1}, f_{2}\right)$ is split with respect to $\left.\Pi_{0}\right)$.

Remark 4.5. In general we can formulate the condition of an integrable system to be split via the Vorobjev data $\left(\Pi_{V e r t}, \Gamma, \mathbb{F}\right)$ associated to the Poisson structure. These data are determined in terms of the Poisson fibration over a symplectic leaf. This is the content of a common project with Camille Laurent-Gengoux [36].

4.4. An action-angle theorem for Poisson manifolds. In this section we include an action-angle theorem in the Poisson context. The contents of this subsection are joint work with Camille Laurent-Gengoux and Pol Vanhaecke and are included in [37].

In this subsection we will impose the following assumptions on our integrable system:

(1) The mapping $\mathbf{F}=\left(f_{1}, \ldots, f_{s}\right)$ defines an integrable system on the Poisson manifold $(M, \Pi)$ of dimension $n$ and (maximal) rank $2 r$.

(2) Suppose that $m \in M$ is a point such that it is regular for the integrable system and the Poisson structure. 
(3) Assume further than the integral manifold $\mathcal{F}_{m}$ of the foliation $X_{f_{1}}, \ldots X_{f_{s}}$ through $m$ is compact (Liouville torus).

Under these conditions we can prove,

Theorem 4.6 (Laurent, Miranda, Vanhaecke). Then there exists $\mathbf{R}$-valued smooth functions $\left(\sigma_{1}, \ldots, \sigma_{s}\right)$ and $\mathbf{R} / \mathbf{Z}$-valued smooth functions $\left(\theta_{1}, \ldots, \theta_{r}\right)$, defined in a neighborhood $U$ of $\mathcal{F}_{m}$ such that

(1) The functions $\left(\theta_{1}, \ldots, \theta_{r}, \sigma_{1}, \ldots, \sigma_{s}\right)$ define a diffeomorphism $U \simeq \mathbf{T}^{r} \times B^{s}$;

(2) The Poisson structure can be written in terms of these coordinates as

$$
\Pi=\sum_{i=1}^{r} \frac{\partial}{\partial \theta_{i}} \wedge \frac{\partial}{\partial \sigma_{i}},
$$

in particular the functions $\sigma_{r+1}, \ldots, \sigma_{s}$ are Casimirs of $\Pi$ (restricted to $U$ );

(3) The leaves of the surjective submersion $\mathbf{F}=\left(f_{1}, \ldots, f_{s}\right)$ are given by the projection onto the second component $\mathbf{T}^{r} \times B^{s}$, in particular, the functions $\sigma_{1}, \ldots, \sigma_{s}$ depend only on the functions $f_{1}, \ldots, f_{s}$.

The proof follows the spirit of Duistermaat in the symplectic case. The steps of the proof are the following:

(1) Topology of the foliation. We first study the topology of the fibration: The fibration in a neighbourhood of a compact connected fiber is a trivial fibration by compact fibers.

(2) These compact fibers are tori: We can recover a $\mathbb{T}^{n}$-action tangent to the leaves of the foliation. This implies a process of uniformization of periods.

$$
\begin{aligned}
\Phi: \mathbf{R}^{r} \times\left(\mathbf{T}^{r} \times B^{s}\right) & \rightarrow \mathbf{T}^{r} \times B^{s} \\
\left(\left(t_{1}, \ldots, t_{r}\right), m\right) & \mapsto \Phi_{t_{1}}^{(1)} \cdots \cdots \Phi_{t_{r}}^{(r)}(m) .
\end{aligned}
$$

(3) We prove that this action is Poisson (we use the fact that if $Y$ is a complete vector field of period 1 and $P$ is a bivector field for which $\mathcal{L}_{Y}^{2} P=0$, then $\left.\mathcal{L}_{Y} P=0\right)$.

(4) Finally we use the Poisson cohomology of the manifold and averaging with respect to this action to check that the action is indeed Hamiltonian.

(5) To construct action-angle coordinates we use Darboux-Carathéodory and the constructed Hamiltonian action of $\mathbb{T}^{n}$ in order to drag normal forms from a neighbourhood of a point to a neighbourhood of a fiber.

In [37] we also give a version for the non-commutative case.

\subsection{Equivariant theorems for Poisson manifolds and rigidity phenomena.} Integrable systems give examples of abelian group actions on Poisson manifolds. In this short paragraph we recall very quickly some equivariant results for compact group actions on Poisson manifolds which preserve the Poisson structure.

For a special kind of Poisson structures called tame, we have the following theorem (see [59]) which is an equivariant version of Weinstein's splitting theorem [75].

Theorem 4.7 (Miranda-Zung [59]). Let $\left(P^{n}, \Pi\right)$ be a smooth Poisson manifold, $p$ a point of $P, 2 k=\operatorname{rank} \Pi(p)$, and $G$ a compact Lie group which acts on $P$ in such 
a way that the action preserves $\Pi$ and fixes the point $p$. Assume that the Poisson structure $\Pi$ is tame at $p$. Then there is a smooth canonical local coordinate system $\left(x_{1}, y_{1}, \ldots, x_{k}, y_{k}, z_{1}, \ldots, z_{n-2 k}\right)$ near $p$, in which the Poisson structure $\Pi$ can be written as

$$
\Pi=\sum_{i=1}^{k} \frac{\partial}{\partial x_{i}} \wedge \frac{\partial}{\partial y_{i}}+\sum_{i j} f_{i j}(z) \frac{\partial}{\partial z_{i}} \wedge \frac{\partial}{\partial z_{j}},
$$

with $f_{i j}(0)=0$, and in which the action of $G$ is linear and preserves the subspaces $\left\{x_{1}=y_{1}=\ldots x_{k}=y_{k}=0\right\}$ and $\left\{z_{1}=\ldots=z_{n-2 k}=0\right\}$.

This result implies local rigidity for compact Poisson group actions.

By using Conn's linearization theorem [14] for semisimple Lie algebra's of compact type, we can also prove an equivariant linearization theorem which can be found in [59].

Among the set of Poisson actions there is a particular class of Poisson actions which deserves a special attention: The class of Hamiltonian actions.

Recently we have proved a rigidity result for Poisson actions with Philippe Monnier and Nguyen Tien Zung.

Not to distract the reader with many technical details, we give a "sloppy" statement here. The exact statement can be found in [56].

Theorem 4.8 (Miranda-Monnier-Zung [56]). Let $\rho_{0}$ and $\rho_{1}$ two close Hamiltonian actions of compact semisimple type on a compact Poisson manifold, then they are equivalent.

In this paper, we also find an application to prove an equivariant normal form result for Poisson structures without assuming that the Poisson structure is tame.

\section{REFERENCES}

[1] A. Albouy, Projective dynamics and classical gravitation Regular and Chaotic Dynamics, 13 (2008) pp. 525-542.

[2] V. I. Arnold, Mathematical methods of classical mechanics. Graduate Texts in Mathematics, 60.

[3] A. Banyaga, The geometry surrounding the Arnold-Liouville Theorem in Advances in geometry, Progress in Mathematics, 172, Birkhauser, 1999.

[4] A. Banyaga and P. Molino, Géométrie des formes de contact complètement intégrables de type torique,, Séminaire Gaston Darboux de Géométrie et Topologie Différentielle, 19911992(Montpellier), Univ. Montpellier II, Montpellier, 1993.

[5] A. V. Bolsinov, B. Jovanovic, Non-commutative integrability, moment map and geodesic flows, Annals of Global Analysis and Geometry 23 (2003), no. 4, 305-322.

[6] A. V. Bolsinov, V.S. Matveev, Singularities of momentum maps of integrable Hamiltonian systems with two degrees of freedom. (Russian summary) Zap. Nauchn. Sem. S.-Peterburg. Otdel. Mat. Inst. Steklov. (POMI) 235 (1996), Differ. Geom. Gruppy Li i Mekh. 15-2, 54-86, 304; translation in J. Math. Sci. (New York) 94 (1999), no. 4, 1477-1500.

[7] Y. Colin de Verdière, Singular Lagrangian manifolds and semiclassical analysis. Duke Math. J. 116 (2003), no. 2, 263-298.

[8] Y. Colin de Verdière and J. Vey, Le lemme de Morse isochore., Topology 18 (1979), 283-293.

[9] Y. Colin de Verdière and S. Vu-Ngoc, Singular Bohr-Sommerfeld rules for 2D integrable systems, Ann. Scient. Éc. Norm. Sup. 36, 2, 1-55, (2003).

[10] C. Curras-Bosch and E. Miranda, Symplectic linearization of singular Lagrangian foliations in $M^{4}$, Differential Geom. Appl. 18 (2003), no. 2 , 195-205. 
[11] M. Chaperon, Normalisation of the smooth focus-focus: a simple proof., 5 pages, preprint 2012.

[12] M. Chaperon, Quelques outils de la théorie des actions différentiables, in: Third Schnepfenried Geometry Conference, vol. 1 ,Schnepfenried, 1982, in: Astérisque, vols. 107-108, Soc. Math. France, Paris, 1983, pp. 259-275.

[13] C. Chevalley and S. Eilenberg, Cohomology theory of Lie groups and Lie algebras, Trans. Amer. Math. Soc. 63 (1948), 85-124.

[14] J. Conn, Normal forms for smooth Poisson structures, Ann. of Math. (2) 121 (1985), no. 3, $565-593$.

[15] T. Delzant Hamiltoniens périodiques et images convexes de l'application moment. Bull. Soc. Math. France 116 (1988), no. 3, 315-339.

[16] B. Khesin and S. Tabaschnikov, Contact complete integrability, with B. Khesin. Reg. Chaotic Dynamics, 15 (2010), 504-520

[17] J.P. Dufour, P. Molino and A. Toulet, Classification des systémes intégrables en dimension 2 et invariants des modèles de Fomenko. C. R. Acad. Sci. Paris Ser. I Math. 318 (1994), no. 10, 949-952.

[18] J.-P. Dufour and P. Molino, Compactification d'actions de $\mathbb{R}^{n}$ et variables action-angle avec singularités, Symplectic geometry, groupoids, and integrable systems (Berkeley, CA, 1989), Springer, New York, 1991, pp. 151-167.

[19] J.J. Duistermaat, On global action-angle coordinates. Comm. Pure Appl. Math. 33 (1980), no. $6,687-706$.

[20] L. H. Eliasson, Normal forms for Hamiltonian systems with Poisson commuting integrals, Ph.D. Thesis (1984).

[21] L.H. Eliasson, Normal forms for Hamiltonian systems with Poisson commuting integralselliptic case, Comment. Math. Helv. 65 (1990), no. 1, 4-35.

[22] Fomenko, Topological classification of all integrable Hamiltonian systems of general types with two degrees of freedom, MSRI Publ., Vol 22 (1991) 131-340.

[23] G. Geiges, Contact Geometry, in: Handbook of Differential Geometry vol. 2 (F.J.E. Dillen and L.C.A. Verstraelen, eds.), to appear.

[24] J.W. Gray, Some global properties of contact structures, Annals of Math. (2), 69, 1959, $421-450$.

[25] V. Guillemin and S. Sternberg, Remarks on a paper of Hermann, Trans. Amer. Math. Soc. 130 (1968) 110-116.

[26] V. Guillemin, D. Shaeffer, On a certain class of Fuchsian partial differential equations. Duke Math. J. 44 (1977), no. 1, 157-199.

[27] V. Guillemin and S. Sternberg, The Gel'fand-Cetlin system and quantization of the complex flag manifolds, J. Funct. Anal., 52(1):106-128, 1983.

[28] V. Guillemin, E. Miranda and A. R. Pires, Codimension one symplectic foliations and regular Poisson manifolds, Bull Braz Math Soc, New Series 42(4), 607-623, 2011.

[29] V. Guillemin, E. Miranda and A. R. Pires, Symplectic and Poisson geometry on b-manifolds arXiv: 1206.2020 .

[30] M. Hamilton and E. Miranda, Geometric quantization of integrable systems with hyperbolic singularities, Annales de l'Institut Fourier, vol. 60 (1) p. 51-85, 2010.

[31] V. Ginzburg, V. Guillemin and Y. Karshon, Moment maps, cobordisms, and Hamiltonian group actions, AMS, 2004.

[32] H. Ito, Convergence of Birkhoff normal forms for integrable systems, Comment. Math. Helv. 64 (1989), no. 3, 412-461.

[33] H. Ito Action-angle coordinates at singularities for analytic integrable systems., Math. Z. 206 (1991), no. 3, 363-407.

[34] B. Kostant, "On the Definition of Quantization," Géométrie Symplectique et Physique Mathématique, Coll. CNRS, No. 237, Paris, (1975), 187-210.

[35] B. Kruglikov and V. Matveev, Vanishing of the entropy pseudonorm for certain integrable systems. ( Electron. Res. Announc. Amer. Math. Soc. 12 (2006), 19-28.

[36] C. Laurent-Gengoux and E. Miranda, Splitting theorem and integrable systems in Poisson manifolds, in preparation, 2012.

[37] C. Laurent-Gengoux, E. Miranda and P. Vanhaecke, Action-angle coordinates for integrable systems on Poisson manifolds, Int. Math. Res. Not. IMRN 2011, no. 8, 1839-1869.

[38] E. Lerman, , Contact toric manifolds, J. Symplectic Geom. 1 (2003), no. 4, 785-828. 
[39] E. Lerman and S. Tolman, Hamiltonian torus actions on symplectic orbifolds and toric varieties. Trans. Amer. Math. Soc. 349 (1997), no. 10, 4201-4230.

[40] P. Libermann, Legendre foliations on contact manifolds. Differential Geom. Appl. 1 (1991), no. $1,57-76$.

[41] P. Libermann, C.M Marle, Symplectic geometry and analytical mechanics. Mathematics and its Applications, 35. D. Reidel Publishing Co., Dordrecht, 1987.

[42] J. Liouville, Note sur l'intégration des équations differentielles de la dynamique, présentée au bureau des longitudes le 29 juin 1853, Journal de Mathématiques pures et appliquées 20 (1855), 137-138.

[43] R. Lutz, Sur la gémétrie des structures de contact invariantes, Ann.Inst. Fourier, 29, 1 (1979), 283-306.

[44] J. Martinet, Formes de contact sur les variétés de dimension 3, Springer LNM, 209 (1971),142-163.

[45] V. Matveev, Integrable Hamiltonian systems with two degrees of freedom. Topological structure of saturated neighborhoods of points of focus-focus and saddle-saddle types. (Russian. Russian summary) Mat. Sb. 187 (1996), no. 4, 29-58; translation in Sb. Math. 187 (1996), no. $4,495-524$.

[46] H. Mineur, Reduction des systèmes mecaniques à $n$ degrées de liberte admettant $n$ intégrales premieres uniformes en involution aux système à variable separees., J. Math Pure Appl., IX Ser. 15 (1936), 385-389.

[47] H. Mineur, Sur les systèmes mécaniques dans lesquels figurent des paramètres fonctions du temps. Étude des systèmes admettant $n$ intégrales premieres uniformes en involution. Extension à ces systèmes des conditions de quantification de Bohr-Sommerfeld., Journal de l'Ecole Polytechnique, Série III, 143ème année (1937), 173-191 and 237-270.

[48] E. Miranda, On the symplectic classification of singular Lagrangian foliations. Proceedings of the IX Fall Workshop on Geometry and Physics (Vilanova i la Geltr£, 2000), 239-244, Publ. R. Soc. Mat. Esp., 3, R. Soc. Mat. Esp., Madrid, 2001.

[49] E. Miranda, On symplectic linearization of singular Lagrangian foliations, Ph. D. thesis, Universitat de Barcelona, 2003, ISBN: 9788469412374.

[50] E. Miranda, A normal form theorem for integrable systems on contact manifolds, Proceedings of the XIII Fall Workshop on Geometry and Physics, Publ. R. Soc. Mat. Esp., 9, R. Soc. Mat. Esp., (2005), 240-246.

[51] E. Miranda, Some rigidity results for Symplectic and Poisson group actions, XV International Workshop on Geometry and Physics, 177-183, Publ. R. Soc. Mat. Esp., 11, R. Soc. Mat. Esp., Madrid, 2007.

[52] E. Miranda, Symmetries and singularities in Hamiltonian systems, J. Phys.: Conf. Ser. 175 012011 doi:10.1088/1742-6596/175/1/012011

[53] E. Miranda, From action-angle coordinates to geometric quantization: a round trip, Oberwolfach report, Geometric quantization in the non-compact setting, 2011.

[54] E. Miranda, Symplectic equivalence of non-degenerate integrable systems, in preparation, 2012.

[55] E. Miranda, Symplectic linearization of semisimple Lie algebra actions, preprint 2011.

[56] E. Miranda, P. Monnier and N.T.Zung, Rigidity of Hamiltonian actions on Poisson manifolds, Adv. Math. 229 (2012), no. 2, 1136-1179.

[57] E. Miranda and S. Vu Ngoc, A singular Poincaré Lemma, IMRN, n 1, 27-46, 2005.

[58] E. Miranda and Nguyen Tien Zung, Equivariant normal forms for nondegenerate singular orbits of integrable Hamiltonian systems,Ann. Sci. Ecole Norm. Sup.,37 (2004), no. 6, 819-839 2004.

[59] E. Miranda and N. T. Zung, A note on equivariant normal forms of Poisson structures, Math. Research Notes, 2006, vol 13-6, 1001-1012.

[60] R. Nest and B. Tsygan, Formal deformations of symplectic manifolds with boundary, J. Reine Angew. Math. 481, 1996, pp. 27-54.

[61] R. Palais, On the existence of slices for actions of non-compact Lie groups. Ann. of Math. 2 73, 1961.

[62] R. Palais, Equivalence of nearby differentiable actions of a compact group. Bull. Amer. Math. Soc. $671961362-364$

[63] G. Paternain, On the topology of manifolds with completely integrable geodesic flows. II , Journ. Geom. Phys. 123 (1994), 289-298. 
[64] A. Pelayo, S.V. Ngoc, Constructing integrable systems of semitoric type. Acta Math. 206 (2011), no. 1, 93-125.

[65] A. Pelayo, S.V. Ngoc, Semitoric integrable systems on symplectic 4-manifolds. Invent. Math. 177 (2009), no. 3, 57-97.

[66] J. Śniatycki, On Cohomology Groups Appearing in Geometric Quantization, Differential Geometric Methods in Mathematical Physics (1975).

[67] A. Toulet, Classification des systèmes integrables en dimension 2. Thèse, Université de Montpellier II, 1996.100 (1978), no. 3, 591-614.

[68] J. Vey, Sur le lemme de Morse. Invent. Math. 40 (1977), no. 1, 1-9.

[69] J. Vey, Sur certains systèmes dynamiques séparables, Amer. J. Math. 100 (1978), 3, 591-614.

[70] S. Vu Ngoc, Formes normales semi-classiques des systèmes complètement intégrables au voisinage d'un point critique de l'application moment. Asymptot. Anal. 24 (2000), no. 3-4, $319-342$.

[71] S. Vu Ngoc, On semi-global invariants for focus-focus singularities. Topology 42 (2003), no. $2,365-380$.

[72] S. Vu Ngoc and C. Wacheux, Smooth normal forms for integrable hamiltonian systems near a focus-focus singularity, http://arxiv.org/abs/1103.3282 .

[73] N.M.J. Woodhouse, Geometric quantization, second edition. Oxford Mathematical Monographs, Oxford Science Publications, The Clarendon Press, Oxford University Press, New York, 1992

[74] A. Weinstein, Lectures on symplectic manifolds. Regional Conference Series in Mathematics, No.29. American Mathematical Society, Providence, R.I., 1977.

[75] A. Weinstein, The local structure of Poisson manifolds., J. Differential Geom. 18 (1983), no. $3,523-557$.

[76] J. Williamson, On the algebraic problem concerning the normal forms of linear dynamical systems, Amer. J. Math. 58:1 (1936), 141-163.

[77] Nguyen Tien Zung, Symplectic topology of integrable Hamiltonian systems. I: ArnoldLiouville with singularities., Compos. Math. 101 (1996), no. 2, 179-215.

[78] Nguyen Tien Zung, Another note on focus-focus singularities. Lett. Math. Phys. 60 (2002), no. $1,87-99$.

[79] Nguyen Tien Zung, Symplectic topology of integrable Hamiltonian systems, II. Compositio Mathematica,138, pp 125-156, 2003.

Departament de Matemàtica Aplicada I, Universitat Politècnica de Catalunya, Barcelona, SPAIN

E-mail address: eva.miranda@upc.edu 